\title{
SMALL POINTS ON SUBVARIETIES OF A TORUS
}

FRANCESCO AMOROSO and EVELINA VIADA

\begin{abstract}
Let $V$ be a subvariety of a torus defined over the algebraic numbers. We give a qualitative and quantitative description of the set of points of $V$ of height bounded by invariants associated to any variety containing $V$. Especially, we determine whether such a set is or is not dense in $V$. We then prove that these sets can always be written as the intersection of $V$ with a finite union of translates of tori of which we control the sum of the degrees.

As a consequence, we prove a conjecture by the first author and David up to a logarithmic factor.
\end{abstract}

\section{Introduction}

In this article, we study the distribution of the small points on varieties over $\overline{\mathbb{Q}}$ imbedded in the torus $\mathbb{G}_{\mathrm{m}}^{n}$ with $n \geq 2$. To simplify the presentation, we fix the usual embedding of $\mathbb{G}_{\mathrm{m}}^{n}$ in $\mathbb{P}^{n}$ given by $\left(x_{1}, \ldots, x_{n}\right) \rightarrow\left(1: x_{1}: \cdots: x_{n}\right)$. A variety $V \subseteq \mathbb{G}_{\mathrm{m}}^{n}$ is the intersection of $\mathbb{G}_{\mathrm{m}}^{n}$ with a variety of $\mathbb{P}^{n}$ defined over $\overline{\mathbb{Q}}$. Note that the varieties that appear in this article are not necessarily irreducible or equidimensional, but they are all defined over $\overline{\mathbb{Q}}$.

We say that

- $\quad V$ is torsion if $V$ is the translate of a subtorus by a torsion point;

- $\quad V$ is transverse if $V$ is irreducible and is not contained in any translate of a proper subtorus.

For a set $S \subseteq \mathbb{G}_{\mathrm{m}}^{n}$, we denote by $\bar{S}$ the Zariski closure of $S$ in $\mathbb{G}_{\mathrm{m}}^{n}$. On $\mathbb{P}^{n}$, we consider the Weil logarithmic absolute height, denoted by $h(\cdot)$. For $\theta \geq 0$, we define

$$
S(\theta)=\{\boldsymbol{\alpha} \in S(\overline{\mathbb{Q}}): h(\boldsymbol{\alpha}) \leq \theta\} .
$$

In the present work, we describe $\overline{V(\theta)}$ in a qualitative and quantitative respect, for different positive reals $\theta$ depending on $V$. Among other results, we prove several sharp effective versions of the toric Bogomolov conjecture. Before we present our main result, we give an overview of the developments around this problem.

DUKE MATHEMATICAL JOURNAL

Vol. 150, No. 2, (c) 2009 DOI 10.1215/

Received 22 May 2008. Revision received 5 May 2009.

2000 Mathematics Subject Classification. Primary 11G10; Secondary 11J81, 14G40.

Viada's work supported by Fonds National Suisse.

1 
Assume that $V$ is not a union of torsion varieties. The toric Bogomolov conjecture, nowadays a theorem of Zhang, claims

$$
\hat{\mu}^{\mathrm{ess}}(V)=\inf \{\theta>0: \overline{V(\theta)}=V\}>0 .
$$

Let us introduce other important invariants of a variety $V \subseteq \mathbb{G}_{\mathrm{m}}^{n}$. The degree of a subvariety of $\mathbb{G}_{\mathrm{m}}^{n}$ is the degree of its Zariski closure in $\mathbb{P}^{n}$. The obstruction index $\omega(V)$ is the minimal degree of a hypersurface containing $V$. By a result of M. Chardin [C], for $V$ equidimensional,

$$
\omega(V) \leq n \operatorname{deg}(V)^{1 / \operatorname{codim}(V)} .
$$

Define $\delta(V)$ as the minimal degree $\delta$ such that $V$ is, as a set, the intersection of hypersurfaces of degree at most $\delta$. Finally, define $\delta_{0}(V)$ as the minimal degree $\delta_{0}$ such that there exists an intersection $X$ of hypersurfaces of degree at most $\delta_{0}$ such that any irreducible component of $V$ is a component of $X$. If $V$ is equidimensional, then

$$
\omega(V) \leq \delta_{0}(V) \leq \delta(V) \leq \operatorname{deg}(V) \leq \delta_{0}(V)^{\operatorname{codim}(V)} .
$$

The first three inequalities are immediate. The last one follows from [P2, Corollary 5, p. 357] (with $m=n, S=\mathbb{P}^{n}$, and $\delta=\delta_{0}(V)$ ).

Let $V$ be a transverse subvariety of $\mathbb{G}_{\mathrm{m}}^{n}$. In [AD1], the first author and David conjecture

$$
\hat{\mu}^{\mathrm{ess}}(V) \geq c(n) \omega(V)^{-1}
$$

for some $c(n)>0$. In [AD1, Theorem 1.4], they prove

$$
\hat{\mu}^{\mathrm{ess}}(V) \geq c(n) \omega(V)^{-1}\left(\log (3 \omega(V))^{-\lambda(\operatorname{codim}(V))},\right.
$$

where $\lambda(k)=\left(9(3 k)^{k+1}\right)^{k}$.

Their proof is long and involved. Mainly, they need an intricate descent argument, hard to read by non specialists. This descent has been used in several occasions by other authors. Our first achievement (Corollary 2.3) is a simple and short proof of a sharp version of [AD1, Theorem 1.4] just mentioned.

Following [BZ], we define $V^{0}$ as the complement in $V$ of the union of all translates of subgroups of positive dimension contained in $V$. Bombieri and Zannier [BZ] and Schmidt $[\mathrm{S}]$ prove that, outside a finite set, the height on $V^{0}(\overline{\mathbb{Q}})$ is bounded from below by a positive value that depends only on the ideal of definition of $V$ and not on the field of definition of $V$. Later, their bound was considerably improved by David and Philippon [DP]. They consider an irreducible variety $V \subseteq \mathbb{G}_{\mathrm{m}}^{n} \subseteq\left(\mathbb{P}^{1}\right)^{n} \subseteq \mathbb{P}^{2^{n}-1}$. 
Let

$$
q=\left(2^{n+4 \operatorname{dim}(V)+22} \operatorname{deg}(V)(\log (\operatorname{deg}(V)+1))^{2 / 3}\right)^{7^{\operatorname{dim}(V)}}
$$

(where $\operatorname{deg}(V)$ is the degree of the Zariski closure of $V$ in $\mathbb{P}^{2^{n}-1}$ ). David and Philippon prove that the set $V\left(q^{-3 / 4}\right)$ is contained in a finite union of translates $B_{j}$ of tori such that $B_{j} \subseteq V$ and $\sum \operatorname{deg}\left(B_{j}\right) \leq q$.

In [AD2], the following lower bound is conjectured.

CONJECTURE 1.1

Let $V \subseteq \mathbb{G}_{\mathrm{m}}^{n}$ be an irreducible variety. There exists $c(n)>0$ such that, for all but finitely many $\boldsymbol{\alpha} \in V^{0}(\overline{\mathbb{Q}})$,

$$
h(\boldsymbol{\alpha}) \geq c(n) \delta(V)^{-1} .
$$

More precisely, there exist $c_{1}(n), c_{2}(n)>0$ and $l \in \mathbb{N}$ such that

$$
V\left(c_{1}(n) \delta(V)^{-1}\right) \subseteq \bigcup_{j=1}^{l} B_{j},
$$

where the $B_{j} \subseteq V$ are translates of tori and

$$
\sum_{j=1}^{l} \operatorname{deg}\left(B_{j}\right) \leq c_{2}(n) \delta(V)^{n} .
$$

From a variant of [AD1, Theorem 1.4], the first author and David deduced a bound of the type (1.4) up to a logarithmic factor. More precisely, in [AD2] the authors defined $\delta(V)$ as the minimal degree $\delta$ such that $V$ is, as a set, a component of the intersection of hypersurfaces of degree at most $\delta$. Note that their definition of $\delta(V)$ coincides with our definition of $\delta_{0}(V)$. In [AD2, Theorem 1.5], they claimed that, according to their notation, for all but finitely many $\boldsymbol{\alpha} \in V^{0}(\overline{\mathbb{Q}})$,

$$
h(\boldsymbol{\alpha}) \geq c(n) \delta(V)^{-1}(\log (3 \delta(V)))^{-\lambda(n-1)},
$$

where $c(n)>0$ and $\lambda(k)=\left(9(3 k)^{(k+1)}\right)^{k}$. We take the opportunity to mention here an error in their approach. Using their definition of $\delta(V)$, at [AD2, p. 561, point (a)] they cannot ensure that $V^{\prime}$ is incompletely defined by forms of degree at most $n D \delta(V)$. The problem is the following. Let $V$ be incompletely defined by forms of degree at most $\delta$, and let $Z$ be a hypersurface of degree at most $\delta$ not containing $V$. Then an irreducible component of $V \cap Z$ is not a priori incompletely defined by forms of degree at most $\delta$. Their proof can be corrected by defining $\delta(V)$ as we have done here. 
The method of [AD2] cannot produce a bound for the sum of the degrees of the translates. A close inspection of their proof shows that one can only bound the degree

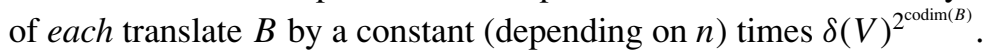

The main result of this article provides a complete description of the points of a variety $V$ of height bounded by different invariants. Let $V \subsetneq \mathbb{G}_{\mathrm{m}}^{n}$ be a variety of codimension $k$. We define

$$
\theta(V)=\delta(V)\left(200 n^{5} \log \left(n^{2} \delta(V)\right)\right)^{(n-k) n(n-1)} .
$$

We decompose $V$ as a (reduced) union $X_{k} \cup \cdots \cup X_{n}$, where $X_{j}$ is an equidimensional variety of codimension $j$. We allow the empty set as an equidimensional variety of arbitrary codimension with no components and degree zero. Our main theorem is the following.

THEOREM 1.2

Let $V=X_{k} \cup \cdots \cup X_{n}$ be as before, and let $\theta=\theta(V)$ be as in (1.5). Then

$$
\overline{V\left(\theta^{-1}\right)}=G_{k} \cup \cdots \cup G_{n}
$$

where $G_{j}$ is either the empty set or a finite union of translates $B_{j, i}$ of tori of codimension $j$ such that $\delta_{0}\left(B_{j, i}\right) \leq \theta$. Moreover, for $r=k, \ldots, n$,

$$
\sum_{i=k}^{r} \theta^{r-i} \operatorname{deg} G_{i} \leq \sum_{i=k}^{r} \theta^{r-i} \operatorname{deg} X_{i} \leq \theta^{r}
$$

The proof is based on a new induction that is simple and optimal. (For more details on the structure of the proof, see Section 2.) Theorem 1.2 has interesting consequences. First, it immediately implies Conjecture 1.1, up to a logarithmic factor. Especially, for an equidimensional $V$ of dimension $d$, the cardinality of the set $V^{0}\left(\theta^{-1}\right)$ is bounded by $\theta^{d} \operatorname{deg}(V) \leq \theta^{n}$.

Second, it generalizes Conjecture 1.1 to all varieties, not only irreducible or equidimensional ones.

A nice feature of Theorem 1.2 is that it provides a complete description of $V\left(\theta(W)^{-1}\right)$ for $\theta(W)$ associated to any variety $W$ containing $V$. More precisely, we have the following.

COROLLARY 1.3

Let $V \subseteq W$ be subvarieties of $\mathbb{G}_{\mathrm{m}}^{n}$. Let $\theta(W)$ be as in (1.5). Then

$$
V\left(\theta(W)^{-1}\right) \subseteq \bigcup B_{j}
$$


where the $B_{j} \subseteq W$ are translates of tori such that $\delta_{0}\left(B_{j}\right) \leq \theta(W)$ and

$$
\sum_{j} \operatorname{deg}\left(B_{j}\right) \leq \theta(W)^{n}
$$

As a consequence, if there exists a component of $V$ which is not contained in any translate $B \subseteq W$ with $\delta_{0}(B) \leq \theta$, then $V\left(\theta(W)^{-1}\right)$ is not dense in $V$.

In other words, the distribution of the points on a variety $V$ depends on the varieties that contain $V$. For instance, suppose that $V$ is irreducible. Choosing respectively $W=V, W$ an intersection of hypersurfaces of degree at most $\delta_{0}(V)$ such that $V$ is a component of $W$, and $W$ a hypersurface of degree $\omega(V)$ containing $V$, Corollary 1.3 describes the points of $V$ of height bounded by the inverse of $\delta(V), \delta_{0}(V)$, and $\omega(V)$, up to a remainder term (see Corollaries 5.1 and 5.2). We note that, for transverse varieties, Corollary 1.3 tells us that, for any $W \supseteq V$, the set of points of $V$ of height bounded by the inverse of $\delta(W)$, up to a remainder term, is never dense.

In Section 5, we also clarify the situation with an example that shows that our results are essentially sharp.

Our results have interesting applications.

Bombieri, Masser and Zannier [BMZ] proved that the intersection of a transverse curve $\mathscr{C}$ with the union of all algebraic subgroups of codimension 2 is a finite set. A recent approach to this kind of problem makes use of an effective version of the Bogomolov theorem (see $[\mathrm{V}]$ in the elliptic case and $[\mathrm{H}]$ in the toric case). More precisely, using a bound for the cardinality of the set of small points on $\mathscr{C}$ one can provide a bound for the intersection of $\mathscr{C}$ with a union of translated codimension-two algebraic subgroups (see [H, Section 7] and for the elliptic case [V, Section 14]). In Corollary 2.3, we give an upper bound for the number of points of height essentially bounded by the inverse of $\omega(\mathscr{C})$. Our estimate improves the one used by Habegger. It also suggests a sharp conjecture in the abelian case.

Let $V$ be a subvariety of $\mathbb{G}_{\mathrm{m}}^{n}$. Following Schmidt [S], we denote by $V^{u}$ the union of all torsion varieties contained in $V$. Let $\delta=\delta(V)$, and let $N=\left(\begin{array}{c}n+\delta \\ n\end{array}\right)$. In [S, Theorems 1(ii), 2(iii)], Schmidt proves that $V^{u}$ is a union of

$$
t \leq(2 \delta)^{n}(11 \delta)^{n^{2}} \exp (4 N !)
$$

torsion varieties. Polynomial bounds in $\delta$ are given in [DP], [R] and [AS]. Theorem 1.2 allows us to further improve these results. In Corollary 5.3, we prove

$$
t \leq \delta^{n}\left(200 n^{5} \log \left(n^{2} \delta\right)\right)^{n^{2}(n-1)^{2}} .
$$

In addition, a bound for the cardinality of the set of small points of $V^{0}$ is used in the proof of a quantitative version of the Mordell-Lang plus Bogomolov problem. Let 
$\Gamma$ be a subgroup of $\mathbb{G}_{\mathrm{m}}^{n}$ of finite rank. Let $\varepsilon \geq 0$. We consider the neighborhood of $\Gamma$,

$$
\Gamma_{\varepsilon}=\left\{\boldsymbol{\alpha} \in \mathbb{G}_{\mathrm{m}}^{n}: \boldsymbol{\alpha}=\mathbf{x z} \text { with } \mathbf{x} \in \Gamma \text { and } h(\mathbf{z}) \leq \varepsilon\right\} .
$$

The Mordell-Lang plus Bogomolov theorem (see [Po]) asserts that $V \cap \Gamma_{\varepsilon}$ is contained in a finite union of translates of subtori contained in $V$. Evertse [E] and Rémond [R] give a quantitative version of this result. To estimate the number of "small points" in $V \cap \Gamma_{\varepsilon}$, they need a bound for the cardinality of $V^{0}(C) \cap \Gamma_{\varepsilon}$ for $C \geq 1$.

A first bound for the cardinality of $V^{0}(C) \cap \Gamma$ appears in [S, Theorem 5]. Later, David and Philippon (see [DP, Theorem 1.4]) improve Schmidt's result obtaining

$$
\left|V^{0}(C) \cap \Gamma\right| \leq C^{r} q^{r+1},
$$

where $q$ is as in (1.3). The method of Schmidt can be easily extended to the case $\varepsilon>0$. Using the bound given in Theorem 1.2, we deduce the following.

COROLLARY 1.4

Let $\Gamma$ be a subgroup of $\mathbb{G}_{\mathrm{m}}^{n}$ of finite rank $r$, and let $V \subsetneq \mathbb{G}_{\mathrm{m}}^{n}$ be a subvariety of codimension $k$. As in (1.5), let

$$
\theta(V)=\delta(V)\left(200 n^{5} \log \left(n^{2} \delta(V)\right)\right)^{(n-k) n(n-1)} .
$$

Then for $C \geq 1$ and for any nonnegative $\varepsilon \leq(2 \theta(V))^{-1}$,

$$
\left|V^{0}(C) \cap \Gamma_{\varepsilon}\right| \leq(5 n C)^{r} \theta(V)^{n+r} .
$$

With respect to [DP, Theorem 1.4], our bound improves not only the dependence on $\operatorname{deg}(V)$ but also the dependence on $n$, at least for varieties of large dimension or degree.

In the special case of a linear variety, Corollary 1.4 can be used to improve considerably the upper bound by Evertse, Schlickewei, and Schmidt [ESS] for the number of nondegenerate* solutions of the equation

$$
a_{1} \alpha_{1}+a_{2} \alpha_{2}+\cdots+a_{n} \alpha_{n}=1 \quad \text { with } \alpha \in \Gamma,
$$

where $\left(a_{1}, \ldots, a_{n}\right) \in \mathbb{G}_{\mathrm{m}}^{n}(K)$ and $\Gamma$ is a subgroup of $\mathbb{G}_{\mathrm{m}}^{n}(K)$ of finite rank $r$ ( $K$ any field of characteristic zero). Their bound is $\exp \left((6 n)^{3 n}(r+1)\right)$. Using Corollary 1.4, this can be improved to $(8 n)^{4 n^{4}(n+r+1)}$, saving an exponential (see Theorem 6.2). As an application of this estimate, we also improve of one exponential the result on multiplicities for a simple linear recurrence sequence of [ESS] (see Corollary 6.3).

*A solution is called nondegenerate if no subsum of the left-hand side of (1.7) vanishes. 
In the next section, we detail the structure of the article. In Sections 3 and 4 , we prove the theorems that lead to the proof of Theorem 1.2, and we present their corollaries. In Section 5, we prove our main theorem and its corollaries. In the last section, we discuss some applications to the Mordell-Lang plus Bogomolov problem.

\section{Structure of the article}

The proof of an effective Bogomolov conjecture given in [AD1] is long and technical. It relies on the fact that $V$ is, in some sense, $p$-adically close to $\zeta V$ for all $p$-torsion points $\zeta$. But also all the translates of $V$ by $p$-torsion points are $p$-adically close to each other. This gives a first simplification: we replace the vanishing principle used in [AD1] by a symmetric vanishing principle. For technical reasons, it is more convenient to use an interpolation determinant than an auxiliary function. This is presented in Section 3.1, where we encode the Diophantine information needed for the proof of Theorem 2.2. The main result of Section 3.1 is Proposition 3.2. It gives an inequality involving some parameters, the essential minimum of a subvariety of $\mathbb{G}_{\mathrm{m}}^{n}$ and two Hilbert functions.

The new key idea to decode the Diophantine information is to use sharp estimates for the Hilbert function. The upper bound is a variant of the main result of [C]. It is proved in [AD1, Lemma 2.5]. The lower bound is a deep result of M. Chardin and P. Philippon [CP, Corollary 3]. In Section 3.2, we use these tools to prove the following.

THEOREM 2.1

Let $V$ be an irreducible subvariety of $\mathbb{G}_{\mathrm{m}}^{n}$ of codimension $k$ which is not a translate of a subtorus. Let

$$
\theta_{0}=\delta_{0}(V)\left(27 n^{2} \log \left(n^{2} \delta_{0}(V)\right)\right)^{k n} .
$$

Then $V\left(\theta_{0}^{-1}\right)$ is contained in a hypersurface $Z$ of degree at most $\theta_{0}$ which does not contain $V$. In particular, $V\left(\theta_{0}^{-1}\right) \subseteq V \cap Z \subsetneq V$ and $\hat{\mu}^{\mathrm{ess}}(V) \geq \theta_{0}^{-1}$.

A preliminary version of Theorem 2.1 was proved in [A]. That preprint is superceded by the present article; therefore it will not be published. A priori, it is difficult to compare Theorem 2.1 with [AD1, Theorem 1.4]. On the one hand, in Theorem 2.1 we do not assume that $V$ is transverse but only that $V$ is not a translate of a subtorus. On the other hand, the bound in Theorem 2.1 depends on $\delta_{0}(V)$ which could potentially be equal to the degree of $V$, while

$$
\omega(V) \leq n \operatorname{deg}(V)^{1 / \operatorname{codim}(V)} .
$$


An innovative reduction process, due to the second author and based on Theorem 2.1 applied to each variety involved, allows us to deduce [AD1, Theorem 1.4]. In Section 4, we prove the following more general result.

THEOREM 2.2

Let $V_{0} \subseteq V_{1}$ be subvarieties of $\mathbb{G}_{\mathrm{m}}^{n}$ of codimensions $k_{0}$ and $k_{1}$, respectively. Assume that $V_{0}$ is irreducible. Let

$$
\theta=\delta\left(V_{1}\right)\left(200 n^{5} \log \left(n^{2} \delta\left(V_{1}\right)\right)\right)^{\left(k_{0}-k_{1}+1\right) k_{0} n} .
$$

Then

- $\quad$ either there exists a translate $B$ of a subtorus such that $V_{0} \subseteq B \subseteq V_{1}$ and $\delta_{0}(B) \leq \theta$,

- $\quad$ or there exists a hypersurface $Z$ of degree at most $\theta$ such that $V_{0} \nsubseteq Z$ and $V_{0}\left(\theta^{-1}\right) \subseteq Z$. Then $V_{0}\left(\theta^{-1}\right) \subseteq V_{0} \cap Z \subsetneq V_{0}$ and clearly $\hat{\mu}^{\text {ess }}\left(V_{0}\right) \geq \theta^{-1}$.

This result has remarkable consequences. The most immediate corollary is an improved and explicit version of [AD1, Theorem 1.4].

COROLLARY 2.3

Let $V \subseteq \mathbb{G}_{\mathrm{m}}^{n}$ be an irreducible variety of codimension $k$. Assume that $V$ is not contained in any translate $B$ of a proper subtorus with $\delta_{0}(B) \leq \theta$ for

$$
\theta=\omega(V)\left(200 n^{5} \log \left(n^{2} \omega(V)\right)\right)^{k^{2} n} .
$$

Then $V\left(\theta^{-1}\right)$ is contained in a hypersurface $Z$ of degree at most $\theta$ such that $V \nsubseteq Z$. As a consequence, we have $\hat{\mu}^{\mathrm{ess}}(V) \geq \theta^{-1}$ for a transverse $V$ and

$$
\left|\mathscr{C}\left(\theta^{-1}\right)\right| \leq \theta \operatorname{deg} \mathscr{C}
$$

for a transverse curve $\mathscr{C}$.

Proof

By definition of $\omega(V)$, there exists an irreducible hypersurface $W$ of degree $\omega(V)$ containing $V$. As $W$ is a hypersurface, $\delta(W)=\operatorname{deg} W=\omega(V)$. Apply Theorem 2.2 with $V_{0}=V, V_{1}=W, k_{0}=k$, and $k_{1}=1$. Then $V\left(\theta^{-1}\right)$ is contained in a hypersurface $Z$ of degree at most $\theta$ such that $V \nsubseteq Z$.

We observe that the proof of the main result of [AD1] requires several technical tools. Namely, the absolute Siegel lemma of Zhang (see [DP, Lemme 4.7]) and an involved variant of the zero lemma of Philippon (see [AD1, Theorem 4.2, Corollary 4.4]). The 
final step of their proof is a complicated descent argument. We avoid all these tools, presenting a short proof relying on basic geometric arguments.

Although our main theorem (Theorem 1.2) contains an improved and explicit version of [AD2, Theorem 1.5], we would like to deduce such a corollary as an immediate consequence of Theorem 2.2.

COROLLARY 2.4

Let $V \subseteq \mathbb{G}_{\mathrm{m}}^{n}$ be an irreducible variety of dimension d. Define

$$
\theta=\delta(V)\left(200 n^{5} \log \left(n^{2} \delta(V)\right)\right)^{(d+1) n^{2}} .
$$

Then $\overline{V\left(\theta^{-1}\right)}$ is a finite union of translates $B_{j}$ of subtori with $\delta_{0}\left(B_{j}\right) \leq \theta$.

Proof

Let $V_{0}$ be one of the finitely many irreducible components of $\overline{V\left(\theta^{-1}\right)}$. Then $\overline{V_{0}\left(\theta^{-1}\right)}=V_{0}$. Apply Theorem 2.2 to the component $V_{0}$ and to $V_{1}=V$. We have $k_{0} \leq n$ and $k_{1}=n-d$. Thus $\left(k_{0}-k_{1}+1\right) k_{0} n \leq(d+1) n^{2}$. It follows that $\overline{V_{0}\left(\theta^{-1}\right)}$ is contained in a translate $B$ of a subtorus such that $B \subseteq V$ and $\delta_{0}(B) \leq \theta$. Varing $V_{0}$ over all components of $\overline{V\left(\theta^{-1}\right)}$, we conclude that $\overline{V\left(\theta^{-1}\right)} \subseteq \bigcup B_{j}$ where $B_{j} \subseteq V$ are translates of subtori with $\delta_{0}\left(B_{j}\right) \leq \theta$. Remark 2.5(ii) gives $\overline{V\left(\theta^{-1}\right)}=\bigcup B_{j}$.

A quantitative description of the small points of $V$ arises from a refined induction based on Theorem 2.2, due to the second author. This leads us to the proof of our main theorem, Theorem 1.2 (see Section 5).

We conclude this section by a simple remark which proves useful in Sections 4 and 5. On a translate of a subtorus, the small points are either dense or the empty set.

Remark 2.5

(i) Let $B$ be a translate of a subtorus. Then, for $\varepsilon \geq 0$, either $B(\varepsilon)$ is empty or it is dense in $B$.

(ii) Let $V \subseteq \mathbb{G}_{\mathrm{m}}^{n}$ be an irreducible variety, and let $\varepsilon>0$. Assume that $V(\varepsilon)$ is contained in a finite union of translates of subtori contained in $V$. Then $\overline{V(\varepsilon)}$ is the union of some of these translates.

Proof

We prove the first assertion. If $B(\varepsilon)$ is nonempty, we can choose $\alpha \in B(\varepsilon)$. Then $B=T \boldsymbol{\alpha}$ for $T$ a subtorus. Note that $T(0)$ is the set of torsion points of $T$. Since $T$ is a torus, we have $\overline{T(0)}=T$. As $h(\boldsymbol{\alpha} \zeta)=h(\boldsymbol{\alpha})$ for any torsion point $\zeta \in \mathbb{G}_{\mathrm{m}}^{n}$, we have

$$
\boldsymbol{\alpha} T(0) \subseteq B(\varepsilon) \subseteq B .
$$

This shows that $B(\varepsilon)$ is Zariski dense in $B$. 
We now prove the second assertion. By assumption, $V(\varepsilon)$ is contained in the union of translates of subtori contained in $V$. Among those translates, choose only the translates $B_{1}, \ldots, B_{k}$ which meet $V(\varepsilon)$. Then $V(\varepsilon) \subseteq B_{1} \cup \cdots \cup B_{k}$ and $B_{i}(\varepsilon)$ is nonempty. By part (i), for $i \in\{1, \ldots, k\}$,

$$
B_{i}=\overline{B_{i}(\varepsilon)} \subseteq \overline{V(\varepsilon)} \subseteq \bigcup_{j=1}^{k} B_{j}
$$

\section{Diophantine analysis}

\subsection{Encoding the information}

We denote $\mathbf{x}=\left(x_{0}, \ldots, x_{n}\right)$. Given a multi-index $\lambda=\left(\lambda_{0}, \ldots, \lambda_{n}\right) \in \mathbb{N}^{n+1}$, we define $\mathbf{x}^{\lambda}=x_{0}^{\lambda_{0}} \cdots x_{n}^{\lambda_{n}}$. Let $I \subset \overline{\mathbb{Q}}[\mathbf{x}]$ be a homogeneous reduced ideal. For $v \in \mathbb{N}$, we denote by $H(\overline{\mathbb{Q}}[\mathbf{x}] / I ; v)$ the Hilbert function $\operatorname{dim}[\overline{\mathbb{Q}}[\mathbf{x}] / I]_{v}$. Let $T$ be a positive integer. We denote by $I^{(T)}$ the $T$-symbolic power of $I$ (i.e., the ideal of polynomials vanishing on the variety defined by $I$ with multiplicity at least $T$ ). Let $V$ be a variety of $\mathbb{G}_{\mathrm{m}}^{n}$. Let $I$ be a radical homogeneous ideal in $\overline{\mathbb{Q}}[\mathbf{x}]$ defining a closed subvariety of $\mathbb{P}^{n}$ whose intersection with $\mathbb{G}_{\mathrm{m}}^{n}$ is $V$. By abuse of notation, we set $H(V ; v)=H(\overline{\mathbb{Q}}[\mathbf{x}] / I ; v)$ and $H(V, T ; v)=H\left(\overline{\mathbb{Q}}[\mathbf{x}] / I^{(T)} ; v\right)$.

The following lemma is one of the key argument of our approach.

LEMMA 3.1

Let $v, T$ be positive integers. Let $W=\left\{\boldsymbol{\alpha}_{1}, \ldots, \boldsymbol{\alpha}_{L}\right\} \subseteq \mathbb{G}_{\mathrm{m}}^{n}(\mathbb{C})$ be a finite set, and let $\lambda_{1}, \ldots, \lambda_{L} \in \mathbb{N}^{n+1}$ be multi-indices of weight $v$. Define

$$
T_{0}:=(L-H(W, T ; v)) T .
$$

Then the multihomogeneous polynomial

$$
F\left(\mathbf{x}_{1}, \ldots, \mathbf{x}_{L}\right)=\operatorname{det}\left(\mathbf{x}_{i}^{\lambda_{j}}\right)_{1 \leq i, j \leq L}
$$

vanishes on $\left(\boldsymbol{\alpha}_{1}, \ldots, \boldsymbol{\alpha}_{L}\right) \in W^{L}$ with multiplicity at least $T_{0}$.

Proof

We assume $\lambda_{i} \neq \lambda_{j}$ for $i \neq j$. Otherwise, $F$ is identically zero, and the proof is clear. If $H(W, T ; v) \geq L$, the assertion is obvious. Assume $H(W, T ; v)<L$, and let $L_{0}=L-H(W, T ; v)$. Let $E_{1}, E_{2} \subseteq \overline{\mathbb{Q}}\left[x_{0}, \ldots, x_{n}\right]_{v}$ be, respectively, the vector space generated by $\mathbf{x}^{\lambda_{1}}, \ldots, \mathbf{x}^{\lambda_{L}}$ and the vector space of homogeneous polynomials of 
degree $v$ vanishing on $W$ with multiplicity at least $T$. Then

$$
\begin{aligned}
\operatorname{dim}\left(E_{1}\right) & =L, \\
\operatorname{dim}\left(E_{2}\right) & =\left(\begin{array}{c}
n+v \\
n
\end{array}\right)-H(W, T ; v), \\
\operatorname{dim}\left(E_{1}+E_{2}\right) & \leq\left(\begin{array}{c}
n+v \\
n
\end{array}\right),
\end{aligned}
$$

whence

$$
\begin{aligned}
\operatorname{dim}\left(E_{1} \cap E_{2}\right) & =\operatorname{dim}\left(E_{1}\right)+\operatorname{dim}\left(E_{2}\right)-\operatorname{dim}\left(E_{1}+E_{2}\right) \\
& \geq L-H(W, T ; v)=L_{0} .
\end{aligned}
$$

Thus, there exist $L_{0}$ linearly independent polynomials

$$
G_{1}=\sum_{j=1}^{L} g_{1 j} \mathbf{x}^{\lambda_{j}}, \ldots, G_{L_{0}}=\sum_{j=1}^{L} g_{L_{0} j} \mathbf{x}^{\lambda_{j}}
$$

vanishing on $W$ with multiplicity at least $T$. Without loss of generality, we can assume

$$
\operatorname{det}\left(g_{k, j}\right)_{\substack{1 \leq k \leq L_{0} \\ L-L_{0}<j \leq L}} \neq 0 .
$$

By elementary operations, we replace the last $L_{0}$ columns of the matrix $\left(\mathbf{x}_{i}^{\lambda_{j}}\right)$ by

$$
{ }^{\tau}\left(G_{k}\left(\mathbf{x}_{1}\right), \ldots, G_{k}\left(\mathbf{x}_{L}\right)\right), \quad k=1, \ldots, L_{0} .
$$

Let $F^{\prime}\left(\mathbf{x}_{1}, \ldots, \mathbf{x}_{L}\right)$ be the determinant of the new matrix. Then

$$
F^{\prime}\left(\mathbf{x}_{1}, \ldots, \mathbf{x}_{L}\right)=c F\left(\mathbf{x}_{1}, \ldots, \mathbf{x}_{L}\right)
$$

for some $c \in \mathbb{C}^{*}$. The polynomials $G_{k}$ vanish on $W$ with multiplicity at least $T$. Developing $F^{\prime}\left(\mathbf{x}_{1}, \ldots, \mathbf{x}_{L}\right)$ with respect to the last $L_{0}$ columns, we see that $F^{\prime}\left(\mathbf{x}_{1}, \ldots, \mathbf{x}_{L}\right)$ vanishes on $\left(\boldsymbol{\alpha}_{1}, \ldots, \boldsymbol{\alpha}_{L}\right) \in \mathbb{P}^{n}(\mathbb{C})^{L}$ with multiplicity at least $T_{0}$.

Let $l$ be a positive integer. We denote by $[l]: \mathbb{G}_{\mathrm{m}}^{n} \rightarrow \mathbb{G}_{\mathrm{m}}^{n}, \boldsymbol{\alpha} \mapsto\left(\alpha_{1}^{l}, \ldots, \alpha_{n}^{l}\right)$ the "multiplication by $l$." Let ker[l] be its kernel. The following inequality is the crucial result of this section.

PROPOSITION 3.2

Let $v$ and $T$ be positive integers, and let $p$ be a prime number. Let $V$ be a subvariety of $\mathbb{G}_{\mathrm{m}}^{n}$. Then

$$
\hat{\mu}^{\mathrm{ess}}(V) \geq\left(1-\frac{H(V, T ; v)}{H(\operatorname{ker}[p] \cdot V ; v)}\right) \frac{T \log p}{p v}-\frac{n}{2 v} \log (v+1) .
$$


Proof

Choose any real $\varepsilon$ such that $\varepsilon>\hat{\mu}^{\text {ess }}(V)$. For simplicity, we define $S=V(\varepsilon)$. Then $S$ is Zariski dense in $V$. We consider the (possibly infinite) matrix

$$
\left(\boldsymbol{\beta}^{\lambda}\right)_{\substack{\beta \in \mathbb{N}^{n+1},|\lambda|=v \\ \beta \in \ln [p] S}}
$$

of rank $L=H(\operatorname{ker}[p] \cdot V ; v)$. We select $\boldsymbol{\beta}_{1}, \ldots, \boldsymbol{\beta}_{L} \in \operatorname{ker}[p] \cdot S$ and $\lambda_{1}, \ldots, \lambda_{L}$ with $\left|\lambda_{j}\right|=v$ such that

$$
\operatorname{det}\left(\boldsymbol{\beta}_{i}^{\lambda_{j}}\right)_{i, j=1, \ldots, L} \neq 0 .
$$

Consider $\boldsymbol{\alpha}_{1}, \ldots, \boldsymbol{\alpha}_{L} \in S$ such that $\boldsymbol{\beta}_{j} \in \operatorname{ker}[p] \boldsymbol{\alpha}_{j}$. We set

$$
F\left(\mathbf{x}_{1}, \ldots, \mathbf{x}_{L}\right)=\operatorname{det}\left(\mathbf{x}_{i}^{\lambda_{j}}\right)_{i, j=1, \ldots, L} \in \mathbb{Z}\left[\mathbf{x}_{1}, \ldots, \mathbf{x}_{L}\right] .
$$

It follows that $F\left(\boldsymbol{\beta}_{1}, \ldots, \boldsymbol{\beta}_{L}\right) \neq 0$. By Lemma 3.1, $F$ vanishes on $\left(\boldsymbol{\alpha}_{1}, \ldots, \boldsymbol{\alpha}_{L}\right)$ with multiplicity at least

$$
T_{0}:=\left(L-H\left(\left\{\boldsymbol{\alpha}_{1}, \ldots, \boldsymbol{\alpha}_{L}\right\}, T ; v\right)\right) T \geq(L-H(V, T ; v)) T .
$$

Let $v$ be a place dividing $p$. Recall that the inequality $|1-\zeta|_{v} \leq p^{-1 /(p-1)}$ holds for every $p$ th root of unity $\zeta$. Thus

$$
\left|\boldsymbol{\alpha}_{j, k}-\boldsymbol{\beta}_{j, k}\right|_{v} \leq p^{-1 /(p-1)}\left|\boldsymbol{\alpha}_{j, k}\right|_{v}
$$

for $j=1, \ldots, L$ and $k=1, \ldots, n$. Thus, by Taylor expansion of $F$ around $\left(\boldsymbol{\alpha}_{1}, \ldots, \boldsymbol{\alpha}_{L}\right)$,

$$
\left|F\left(\boldsymbol{\beta}_{1}, \ldots, \boldsymbol{\beta}_{L}\right)\right|_{v} \leq p^{-T_{0} /(p-1)} \prod_{j=1}^{L}\left|\boldsymbol{\alpha}_{j}\right|_{v}^{v},
$$

where $\left|\boldsymbol{\alpha}_{k}\right|_{v}=\max \left\{1,\left|\alpha_{j, 1}\right|_{v}, \ldots,\left|\alpha_{j, n}\right|_{v}\right\}$.

By the ultrametric inequality for $v \nmid \infty$ and by the Hadamard inequality for $v \mid \infty$, we obtain that, for an arbitrary place $v$,

$$
\left|F\left(\boldsymbol{\beta}_{1}, \ldots, \boldsymbol{\beta}_{L}\right)\right|_{v} \leq \begin{cases}\prod_{j=1}^{L}\left|\boldsymbol{\beta}_{j}\right|_{v}^{v} & \text { if } v \nmid \infty, \\ L^{L / 2} \prod_{j=1}^{L}\left|\boldsymbol{\beta}_{j}\right|_{v}^{v} & \text { if } v \mid \infty .\end{cases}
$$

Since $\boldsymbol{\alpha}_{k}$ is a translate of $\boldsymbol{\beta}_{k}$ by a torsion point, $\left|\boldsymbol{\beta}_{k}\right|_{v}=\left|\boldsymbol{\alpha}_{k}\right|_{v}$. We apply the product formula:

$$
0 \leq-\frac{T_{0} \log p}{p-1}+\frac{L}{2} \log L+v \sum_{j=1}^{L} h\left(\boldsymbol{\alpha}_{j}\right) \leq-\frac{T_{0} \log p}{p}+\frac{L}{2} \log L+v L \varepsilon .
$$


Moreover, $L \leq(v+1)^{n}$. Thus

$$
\varepsilon \geq \frac{T_{0} \log p}{\operatorname{Lpv}}-\frac{n}{2 v} \log (v+1) .
$$

Taking the limit for $\varepsilon$ which tends to $\hat{\mu}^{\text {ess }}(V)$, we obtain the wished bound.

\subsection{Decoding the information}

As announced in Section 2, to prove Theorem 2.1 we need an upper bound for the Hilbert function. The next proposition follows from a result of M. Chardin [C].

PROPOSITION 3.3

Let $V \subseteq \mathbb{P}^{n}$ be an irreducible variety of dimension $d$ and codimension $k=n-d$. Let $v$ and $T$ be positive integers. Then

$$
H(V, T ; v) \leq\left(\begin{array}{c}
T-1+k \\
k
\end{array}\right)\left(\begin{array}{c}
v+d \\
d
\end{array}\right) \operatorname{deg}(V) .
$$

Proof

See [AD1, Lemma 2.5].

We also need a sharp lower bound for the Hilbert function. This is a deep result of M. Chardin and P. Philippon.

THEOREM 3.4 ([CP, Corollary 3])

Let $K$ be a field, and let $A=K\left[x_{0}, \ldots, x_{n}\right]$. Let $I, J \subseteq A$ be two homogeneous ideals with $J$ of codimension $r$. Let $d_{1} \geq \cdots \geq d_{m}$ be positive integers. Assume that (i) $\quad I=\left(F_{1}, \ldots, F_{m}\right)$ with $\operatorname{deg} F_{j}=d_{j}$;

(ii) $J$ contains the intersection of the primary components of codimension $r$ of $I$. Then, for $v>d_{1}+\cdots+d_{r}-r$, we have

$$
H(A / J ; v) \geq \operatorname{deg} J \cdot\left(\begin{array}{c}
v+n-\left(d_{1}+\cdots+d_{r}\right) \\
n-r
\end{array}\right) .
$$

As a corollary, we have the following. 
COROLLARY 3.5

Let $V \subseteq \mathbb{P}^{n}$ be an equidimensional variety of dimension d and codimension $k=n-d$. Define $m=k\left(\delta_{0}(V)-1\right)$. Then, for any $v>m$, we have

$$
H(V ; v) \geq\left(\begin{array}{c}
v+d-m \\
d
\end{array}\right) \operatorname{deg}(V) .
$$

Proof

In Theorem 3.4, we choose for $J$ the ideal of definition of $V$, and $r=k$ is the codimension of $V$. Furthermore, we choose for $I$ an ideal defined by forms of degree at most $\delta_{0}(V)$ such that all components of $V$ are components of the zero set of $I$.

Let $V$ be an irreducible variety of $\mathbb{G}_{\mathrm{m}}^{n} \subseteq \mathbb{P}^{n}$, and let $p$ be a prime number. In order to prove Theorem 2.1, we apply Corollary 3.5 to $V^{\prime}=\operatorname{ker}[p] \cdot V$. Therefore, we need an upper bound for $\delta_{0}\left(V^{\prime}\right)$ and a lower bound for $\operatorname{deg}\left(V^{\prime}\right)$. These bounds are the object of Lemma 3.8.

LEMMA 3.6

Let $X_{1}, \ldots, X_{t}$ subvarieties of $\mathbb{G}_{\mathrm{m}}^{n}$. Then $\delta\left(\bigcup_{j} X_{j}\right) \leq \sum_{j} \delta\left(X_{j}\right)$.

Proof

It is enough to prove this lemma with $t=2$. Let $f_{1}, \ldots, f_{a}$ be equations of degree at most $\delta\left(X_{1}\right)$ defining $X_{1}$. Similarly, let $g_{1}, \ldots, g_{b}$ be equations of degree at most $\delta\left(X_{2}\right)$ defining $X_{2}$. Then $X_{1} \cup X_{2}$ is defined by the equations $f_{i} g_{j}$ with $1 \leq i \leq a$ and $1 \leq j \leq b$.

Let $V$ and $X$ be subvarieties of $\mathbb{G}_{\mathrm{m}}^{n}$. Assume that $V$ is irreducible. We say that

- $\quad V$ is imbedded in $X$ if there exists an irreducible component $W$ of $X$ such that $V \subsetneq W$.

In other words, $V$ is imbedded in $X$ if $V \subseteq X$ and $V$ is not a component of $X$.

\section{Remark 3.7}

Let $V$ be irreducible. Assume that $V$ is imbedded in $X$.

(i) Let $X \subseteq X^{\prime}$. Then $V$ is imbedded in $X^{\prime}$.

(ii) Let $\zeta \in \mathbb{G}_{\mathrm{m}}^{n}$. Then $\zeta V$ is imbedded in $\zeta X$.

(iii) Let $X_{1}, \ldots, X_{t}$ be subvarieties of $\mathbb{G}_{\mathrm{m}}^{n}$, and let $V$ be imbedded in $\bigcup_{j} X_{j}$. Then $V$ is imbedded in at least one of the $X_{j}$. 
LEMMA 3.8

Let $V$ be an irreducible subvariety of $\mathbb{G}_{\mathrm{m}}^{n}$. Let $G \subseteq \mathbb{G}_{\mathrm{m}}^{n}$ be a finite group.

(i) There exists a variety $X^{\prime}$ such that

- $\quad V \subseteq X^{\prime}$

- $\delta\left(X^{\prime}\right) \leq \delta_{0}(V)$, and

- $\quad \zeta V$ is a component of $X^{\prime}$ for all $\zeta \in G$ such that $\zeta V \subseteq X^{\prime}$.

(ii)

Let $t$ be the number of irreducible components of $V^{\prime}=G \cdot V$. Then

$$
\operatorname{deg}\left(V^{\prime}\right)=t \operatorname{deg}(V) \quad \text { and } \quad \delta_{0}\left(V^{\prime}\right) \leq t \delta_{0}(V) .
$$

Proof

We prove (i). By definition of $\delta_{0}(V)$, there exists a variety $X$ defined by equations of degree at most $\delta_{0}(V)$ such that $V$ is a component of $X$. Let $S$ be the set of $\zeta \in G$ such that $\zeta V$ is imbedded in $X$. Then $V \subseteq \zeta^{-1} X$. We define

$$
X^{\prime}=X \cap \bigcap_{\zeta \in S} \zeta^{-1} X
$$

Note that $V \subseteq X^{\prime}$. Furthermore, the varieties $X$ and $\zeta^{-1} X$ are intersections of hypersurfaces of degree at most $\delta_{0}(V)$. Thus $\delta\left(X^{\prime}\right) \leq \delta_{0}(V)$.

We show that no translate $\zeta V$ is imbedded in $X^{\prime}$. Assume by contradiction that $\zeta V$ was imbedded in $X^{\prime}$ for some $\zeta \in G$. We prove that $1 \in S$. Then $V$ would be imbedded in $X$, which contradicts the fact that $V$ is a component of $X$. Since $\zeta$ has finite order, to prove $\mathbf{1} \in S$ it is sufficient to prove that $\zeta^{n} \in S$ for all positive integers $n$. We proceed by induction. Since $X^{\prime} \subseteq X, \zeta V$ is imbedded in $X$ and $\zeta \in S$. We now assume that $\zeta^{n} \in S$ for some $n \geq 1$, and we prove that $\zeta^{n+1} \in S$. Since $X^{\prime} \subseteq \zeta^{-n} X$, $\zeta V$ is imbedded in $\zeta^{-n} X$. Thus $\zeta^{n+1} V$ is imbedded in $X$ and $\zeta^{n+1} \in S$.

We now prove (ii). Let $\zeta_{1} V, \ldots, \zeta_{t} V$ be the components of $V^{\prime}$. Clearly, $\operatorname{deg}\left(V^{\prime}\right)=$ $\sum_{j} \operatorname{deg}\left(\zeta_{j} V\right)=t \operatorname{deg}(V)$. Let $j \in\{1, \ldots, t\}$. By part (i) (with $\zeta_{j} V$ instead of $V$ ), we can choose a variety $X_{j}$ such that $\zeta_{j} V \subseteq X_{j}$ and $\delta\left(X_{j}\right) \leq \delta_{0}(V)$. Furthermore, if $\zeta V \subseteq X_{j}$ for some $\zeta \in G$, then $\zeta V$ is a component of $X_{j}$. Thus, in view of Remark 3.7(iii), $\zeta_{1} V, \ldots, \zeta_{t} V$ are components of $X_{1} \cup \cdots \cup X_{t}$. By lemma 3.6,

$$
\delta_{0}\left(V^{\prime}\right) \leq \delta\left(X_{1} \cup \cdots \cup X_{t}\right) \leq t \delta_{0}(V) .
$$

The stabilizer of a variety $V$ is

$$
\operatorname{Stab}(V)=\left\{\boldsymbol{\alpha} \in \mathbb{G}_{\mathrm{m}}^{n}: \boldsymbol{\alpha} V=V\right\} .
$$

We denote by $\operatorname{Stab}(V)^{0}$ the connected component of $\operatorname{Stab}(V)$ through the neutral element. We recall that $\operatorname{dim}(\operatorname{Stab}(V)) \leq \operatorname{dim}(V)$ with equality if and only if $V$ is a 
translate of a subtorus. In addition,

$$
\operatorname{deg}(\operatorname{Stab}(V)) \leq \operatorname{deg}(V) \delta(V)^{\operatorname{dim}(V)} \leq \operatorname{deg}(V)^{\operatorname{dim}(V)+1} .
$$

We also recall the following.

LEMMA 3.9

Let $l$ be an integer coprime with $\left[\operatorname{Stab}(V): \operatorname{Stab}(V)^{0}\right]$. Then $\operatorname{ker}[l] \cdot V$ is a union of $l^{\operatorname{codim}(\operatorname{Stab} V)}$ distinct components (which are translates of $V$ by $l$-torsion points).

All the previous statements concerning stabilizers are proved in [Hi, Lemma 6].

At last, we are ready to prove the main result of this section, Theorem 2.1. For the convenience of the reader, we recall the statement.

THEOREM 2.1

Let $V$ be an irreducible subvariety of $\mathbb{G}_{\mathrm{m}}^{n}$ of codimension $k$ which is not a translate of a subtorus. Let

$$
\theta_{0}=\delta_{0}(V)\left(27 n^{2} \log \left(n^{2} \delta_{0}(V)\right)\right)^{k n} .
$$

Then $V\left(\theta_{0}^{-1}\right)$ is contained in a hypersurface $Z$ of degree at most $\theta_{0}$ which does not contain $V$. In particular, $V\left(\theta_{0}^{-1}\right) \subseteq V \cap Z \subsetneq V$ and $\hat{\mu}^{\mathrm{ess}}(V) \geq \theta_{0}^{-1}$.

Proof

Let $d=n-k=\operatorname{dim}(V)$, and let $\delta_{0}=\delta_{0}(V)$. In the sequel of the proof, we use several times the fact that $n>k \geq 1$. Especially, the inequality $n \geq 2$ allows us to improve numerical constants. Let

$$
N=1.41\left(13 n^{2} \log \left(n^{2} \delta_{0}\right)\right)^{k} .
$$

We remark that $N \geq 1.41 \times 13 \times 4 \times \log (4)>101$. By [RS, Theorems 9, 10], $\sum_{p \leq x} \log p \leq 1.02 x$ for $x \geq 1$, and $\sum_{p \leq x} \log p \geq 0.84 x$ for $x \geq 101$. Thus

$$
\begin{aligned}
\sum_{N / 1.41 \leq p \leq N} \log p & \geq\left(0.84-\frac{1.02}{1.41}\right) N \\
& \geq 0.11 \cdot N \\
& \geq 0.11 \cdot\left(13 n^{2} \log \left(n^{2} \delta_{0}\right)\right)^{k} \\
& \geq 0.11 \cdot 13 n \cdot 2^{k} \log \delta_{0} \\
& >n k \log \delta_{0} .
\end{aligned}
$$


If for any prime $p$ with $N / 1.41 \leq p \leq N$ we have

$$
p \mid\left[\operatorname{Stab}(V): \operatorname{Stab}(V)^{0}\right],
$$

then

$$
\log \left[\operatorname{Stab}(V): \operatorname{Stab}(V)^{0}\right] \geq \sum_{N / 1.41 \leq p \leq N} \log p>n k \log \delta_{0}
$$

This is impossible because, by (3.9) and (1.2),

$$
\left[\operatorname{Stab}(V): \operatorname{Stab}(V)^{0}\right] \leq \operatorname{deg}(\operatorname{Stab}(V)) \leq \operatorname{deg}(V)^{\operatorname{dim}(V)+1} \leq \delta_{0}^{n k} .
$$

We conclude that there exists a prime $p \nmid\left[\operatorname{Stab}(V): \operatorname{Stab}(V)^{0}\right]$ satisfying

$$
\left(13 n^{2} \log \left(n^{2} \delta_{0}\right)\right)^{k} \leq p \leq 1.41\left(13 n^{2} \log \left(n^{2} \delta_{0}\right)\right)^{k} .
$$

Since $p \nmid\left[\operatorname{Stab}(V): \operatorname{Stab}(V)^{0}\right]$, Lemma 3.9 implies that the variety $V^{\prime}=\operatorname{ker}[p] \cdot V$ is a union of $p^{\operatorname{codim}(\operatorname{Stab} V)}$ distinct components that are translates of $V$ by a $p$-torsion point. Since $V$ is not a translate of a subtorus,

$$
k+1 \leq \operatorname{codim}(\operatorname{Stab} V) \leq n .
$$

By Lemma 3.8(ii),

$$
\operatorname{deg}\left(V^{\prime}\right) \geq p^{k+1} \operatorname{deg}(V) \quad \text { and } \quad \delta_{0}\left(V^{\prime}\right) \leq p^{n} \delta_{0} .
$$

We apply Proposition 3.3 to $V$ and Corollary 3.5 to $V^{\prime}$. As in the statement of Corollary 3.5, let $m=k\left(\delta_{0}\left(V^{\prime}\right)-1\right)$. The upper bound for $\delta_{0}\left(V^{\prime}\right)$ in (3.11) gives

$$
m+1 \leq k p^{n} \delta_{0} .
$$

Choose

$$
v=m d+m \quad \text { and } \quad T=\left[0.1 p^{1+1 / k}\right] .
$$

Let $f(n, k)=((n+1-k) k)^{1 /(n k)}$. We have

$$
\frac{\partial f}{\partial k}=-\frac{1}{n k^{2}}\left(\log ((n+1-k) k)+\frac{k}{n+1-k}-1\right)
$$

and $\log ((n+1-k) k)+k /(n+1-k) \geq \log n+1 / n>1$. Thus $k \mapsto f(n, k)$ is a decreasing function and $f(n, k) \leq f(n, 1)=n^{1 / n} \leq 3^{1 / 3}$. By the upper bound for 
$m+1$ and for $p$ (see (3.10)), we obtain

$$
\begin{aligned}
v+1 \leq(d+1)(m+1) & \leq(n+1-k) k p^{n} \delta_{0} \\
& \leq\left(f(n, k) 1.41^{1 / k} 13 n^{2} \log \left(n^{2} \delta_{0}\right)\right)^{k n} \delta_{0} \\
& \leq\left(3^{1 / 3} \cdot 1.41 \cdot 13 n^{2} \log \left(n^{2} \delta_{0}\right)\right)^{k n} \delta_{0} .
\end{aligned}
$$

Note that $3^{1 / 3} \cdot 1.41 \cdot 13<27$. Thus

$$
v+1 \leq\left(27 n^{2} \log \left(n^{2} \delta_{0}\right)\right)^{k n} \delta_{0}=\theta_{0}
$$

and

$$
\theta_{0}^{-1}<v^{-1}
$$

Let $W$ be the Zariski closure of the set $V\left(\theta_{0}^{-1}\right)$, and let $W^{\prime}=\operatorname{ker}[p] \cdot W$. Then

$$
\hat{\mu}^{\mathrm{ess}}(W) \leq \theta_{0}^{-1}<v^{-1} .
$$

Furthermore, as $W \subseteq V$ and $W^{\prime} \subseteq V^{\prime}$,

$$
H(W, T ; \nu) \leq H(V, T ; v) \quad \text { and } \quad H\left(W^{\prime} ; \nu\right) \leq H\left(V^{\prime} ; \nu\right) .
$$

We show that $H\left(W^{\prime} ; v\right)<H\left(V^{\prime} ; v\right)$. Assume by contradiction that

$$
H\left(W^{\prime} ; v\right)=H\left(V^{\prime} ; v\right)
$$

Apply Corollary 3.5 to the variety $V^{\prime}$, and apply Proposition 3.3 to the variety $V$. Then, by the lower bound for $\operatorname{deg}\left(V^{\prime}\right)$ given in (3.11),

$$
\frac{H(W, T ; v)}{H\left(W^{\prime} ; v\right)} \leq \frac{H(V, T ; v)}{H\left(V^{\prime} ; v\right)} \leq \frac{\left(\begin{array}{c}
T-1+k \\
k
\end{array}\right)\left(\begin{array}{c}
v+d \\
d
\end{array}\right) \operatorname{deg}(V)}{\left(\begin{array}{c}
v+d-m \\
d
\end{array}\right) \operatorname{deg}\left(V^{\prime}\right)} \leq \frac{\left(\begin{array}{c}
T-1+k \\
k
\end{array}\right)\left(\begin{array}{c}
v+d \\
d
\end{array}\right)}{\left(\begin{array}{c}
v+d-m \\
d
\end{array}\right) p^{k+1}} .
$$

By the choice $T=\left[0.1 p^{1+1 / k}\right]$, we have $\left(\begin{array}{c}T-1+k \\ k\end{array}\right) \leq T^{k} \leq 0.1 p^{k+1}$. Moreover, by the choice $v=m d+m$,

$$
\left(\begin{array}{c}
v+d \\
d
\end{array}\right)\left(\begin{array}{c}
v+d-m \\
d
\end{array}\right)^{-1}=\prod_{j=1}^{d} \frac{v+j}{v-m+j} \leq\left(1+\frac{m}{v-m}\right)^{d}=\left(1+\frac{1}{d}\right)^{d} \leq e .
$$

Thus

$$
\frac{H(W, T ; \nu)}{H\left(W^{\prime} ; \nu\right)} \leq 0.1 e<0.3
$$


By Proposition 3.2 (with $V$ replaced by $W$ ),

$$
\begin{aligned}
\hat{\mu}^{\mathrm{ess}}(W) & \geq\left(1-\frac{H(W, T ; v)}{H\left(W^{\prime} ; v\right)}\right) \frac{T \log p}{p v}-\frac{n}{2 v} \log (v+1) \\
& \geq\left(\frac{0.7 T \log p}{p}-\frac{n}{2} \log (v+1)\right) v^{-1} .
\end{aligned}
$$

We still need a bound for $0.7 T \log p / p$ and for $(n / 2) \log (v+1)$. By the choice of $T$,

$$
\frac{0.7 T \log p}{p} \geq 0.7\left(0.1 p^{1 / k}-\frac{1}{p}\right) \log p \text {. }
$$

By the lower bound for $p$ in (3.10),

$$
\begin{aligned}
\frac{0.7 T \log p}{p} & \geq 0.7\left(0.1 \cdot 13 n^{2} \log \left(n^{2} \delta_{0}\right)-\frac{1}{13 n^{2}}\right) k \log \left(13 n^{2}\right) \\
& \geq 0.7\left(0.1 \cdot 13-\frac{1}{13 n^{4}}\right) n^{2} \log \left(n^{2} \delta_{0}\right) \cdot k \log \left(13 n^{2}\right) .
\end{aligned}
$$

Since $n \geq 2$, we have

$$
0.7\left(0.1 \cdot 13-\frac{1}{13 n^{4}}\right) \log \left(13 n^{2}\right) \geq 0.7\left(0.1 \cdot 13-\frac{1}{13 \cdot 16}\right) \log (13 \cdot 4)>3.5 .
$$

Thus

$$
\frac{0.7 T \log p}{p} \geq 3.5 k n^{2} \log \left(n^{2} \delta_{0}\right)
$$

Using (3.12), $27 n^{2} \leq 2^{5} n^{2} \leq n^{7}$, and $\log x<x$ for $x>0$, we get

$$
\frac{n}{2} \log (v+1) \leq \frac{n}{2}\left(k n \log \left(n^{7} \cdot n^{2} \delta_{0}\right)+\log \left(\delta_{0}\right)\right) \leq \frac{n}{2} k n \log \left(n^{9} \delta_{0}^{2}\right) .
$$

Thus

$$
\frac{n}{2} \log (v+1) \leq 3 k n^{2} \log \left(n^{2} \delta_{0}\right) .
$$

Replacing (3.16) and (3.17) into (3.15), we get

$$
\hat{\mu}^{\text {ess }}(W) \geq 0.5 k n^{2} \log \left(n^{2} \delta_{0}\right) v^{-1}>v^{-1} .
$$

This contradicts (3.13) and shows that

$$
H\left(W^{\prime} ; v\right)<H\left(V^{\prime} ; v\right) .
$$


Equivalently, there exists a homogeneous polynomial $F$ of degree $v \leq \theta_{0}$ vanishing on $W^{\prime}$ but not on $V^{\prime}$. Replacing $F(\mathbf{x})$ by $F(\zeta \mathbf{x})$ for a suitable $\zeta \in \operatorname{ker}[p]$, we can assume $F \neq 0$ on $V$. (Recall that $W^{\prime}$ is invariant by translation by $p$-torsion points.) Let $Z \subseteq \mathbb{G}_{\mathrm{m}}^{n}$ be the hypersurface defined by $F$. By construction $V_{0}\left(\theta_{0}^{-1}\right) \subseteq W \subseteq W^{\prime} \subseteq Z, V \nsubseteq Z$ and $\operatorname{deg}(Z)=v \leq \theta_{0}$. This proves the theorem.

\section{Qualitative description of the small points}

In this section, we prove Theorem 2.2. For the convenience of the reader, we recall the statement.

THEOREM 2.2

Let $V_{0} \subseteq V_{1}$ be subvarieties of $\mathbb{G}_{\mathrm{m}}^{n}$ of codimensions $k_{0}$ and $k_{1}$, respectively. Assume that $V_{0}$ is irreducible. Let

$$
\theta=\delta\left(V_{1}\right)\left(200 n^{5} \log \left(n^{2} \delta\left(V_{1}\right)\right)\right)^{\left(k_{0}-k_{1}+1\right) k_{0} n} .
$$

Then

- $\quad$ either there exists a translate $B$ of a subtorus such that $V_{0} \subseteq B \subseteq V_{1}$ and $\delta_{0}(B) \leq \theta$,

- $\quad$ or there exists a hypersurface $Z$ of degree at most $\theta$ such that $V_{0} \nsubseteq Z$ and $V_{0}\left(\theta^{-1}\right) \subseteq Z$.

Proof

We simply denote $\delta=\delta\left(V_{1}\right)$. By contradiction, we suppose that the conclusion of Theorem 2.2 does not hold. Thus

$V_{0}$ is not contained in any translate $B \subseteq V_{1}$ of a subtorus with $\delta_{0}(B) \leq \theta$,

and

each hypersurface $Z$ of degree at most $\theta$ such that $V_{0}\left(\theta^{-1}\right) \subseteq Z$ contains $V_{0}$.

For $r \in\left\{0, \ldots, k_{0}-k_{1}+1\right\}$, we define

$$
D_{r}=\delta\left(200 n^{5} \log \left(n^{2} \delta\right)\right)^{r k_{0} n}
$$

Since $r \leq k_{0}-k_{1}+1$, we have $D_{r} \leq \theta$. Using an inductive process on $r$, we are going to construct a chain of varieties

$$
X_{0} \supseteq \cdots \supseteq X_{r} \supseteq X_{r+1} \supseteq \cdots \supseteq X_{k_{0}-k_{1}+1}
$$

satisfying the following. 
CLAIM

We have the following:

(i) $\quad V_{0} \subseteq X_{r}$;

(ii) each irreducible component of $X_{r}$ containing $V_{0}$ has codimension at least $r+k_{1}$

(iii) $\delta\left(X_{r}\right) \leq D_{r}$.

Theorem 2.2 is proved if we show this claim for $r=k_{0}-k_{1}+1$. Indeed, by (i) there exists an irreducible component $W$ of $X_{k_{0}-k_{1}+1}$ which contains $V_{0}$. By (ii), codim $W \geq k_{0}+1$. This gives a contradiction.

We now define $X_{r}$ and prove our claim by induction on $r$.

- For $r=0$, we simply choose $X_{0}=V_{1}$.

- We assume that our claim holds for some $r \in\left\{0, \ldots, k_{0}-k_{1}\right\}$, and we prove that it holds for $r+1$, as well. Let $0 \leq s \leq t$ be integers, and let $W_{1}, \ldots, W_{t}$ be the irreducible components of $X_{r}$ enumerated in such a way that

$$
V_{0} \subseteq W_{j} \text { if and only if } 1 \leq j \leq s .
$$

Since $V_{0} \subseteq X_{r}$, we have $s \geq 1$. Assertion (ii) of our claim for $r$ implies that $r+k_{1} \leq \operatorname{codim}\left(W_{j}\right) \leq k_{0}$ for $j=1, \ldots, s$.

Let $j \in\{1, \ldots, s\}$. Since $\delta\left(X_{r}\right) \leq D_{r}$, the variety $W_{j}$ is an irreducible component of an intersection of hypersurfaces of degree at most $D_{r}$. Thus $\delta_{0}\left(W_{j}\right) \leq D_{r} \leq \theta$. Moreover,

$$
V_{0} \subseteq W_{j} \subseteq X_{r} \subseteq X_{0}=V_{1}
$$

By assumption (4.18), $W_{j}$ is not a translate of a subtorus. Let

$$
\theta_{0}=D_{r}\left(27 n^{2} \log \left(n^{2} D_{r}\right)\right)^{k_{0} n}
$$

Note that $\delta_{0}\left(W_{j}\right)\left(27 n^{2} \log \left(n^{2} \delta_{0}\left(W_{j}\right)\right)\right)^{k n} \leq \theta_{0}$. In view of Theorem 2.1, the set $W_{j}\left(\theta_{0}^{-1}\right)$ is contained in a hypersurface $Z_{j}$ which does not contain $W_{j}$ and such that $\operatorname{deg} Z_{j} \leq \theta_{0}$. For $x>0$, we have $\log x \leq x^{1 / 2}$. Furthermore, $n \geq 2$. Thus

$$
\begin{aligned}
n^{2} D_{r} & =n^{2} \delta\left(200 n^{5} \log \left(n^{2} \delta\right)\right)^{r k_{0} n} \leq n^{2} \delta\left(200 n^{6} \delta^{1 / 2}\right)^{r k_{0} n} \\
& \leq n^{2} \delta\left(200 n^{6} \delta\right)^{n^{3}-1} \leq\left(200 n^{6} \delta\right)^{n^{3}} \\
& \leq\left(n^{2} \delta\right)^{7 n^{3}} .
\end{aligned}
$$


(For the last inequalities, use $r k_{0} n \leq(n-1)^{2} n \leq n^{3}-1$ and $200 \leq 2^{8} \leq n^{8}$.) Thus

$$
\begin{aligned}
\theta_{0} & \leq D_{r}\left(27 n^{2} \times 7 n^{3} \log \left(n^{2} \delta\right)\right)^{k_{0} n} \\
& =\delta\left(200 n^{5} \log \left(n^{2} \delta\right)\right)^{r k_{0} n}\left(189 n^{5} \log \left(n^{2} \delta\right)\right)^{k_{0} n} \\
& <D_{r+1} .
\end{aligned}
$$

Since $V_{0} \subseteq W_{j}$,

$$
V_{0}\left(\theta_{0}^{-1}\right) \subseteq W_{j}\left(\theta_{0}^{-1}\right) \subseteq Z_{j}
$$

As $\operatorname{deg} Z_{j} \leq \theta_{0}<D_{r+1} \leq \theta$, relation (4.19) implies that $V_{0} \subseteq Z_{j}$. Thus, for $j=1, \ldots, s$, we have $V_{0} \subseteq Z_{j}$ and

$$
V_{0} \subseteq \bigcap_{j=1}^{s} Z_{j} .
$$

Let

$$
X_{r+1}=X_{r} \cap Z_{1} \cap \cdots \cap Z_{s}
$$

Then $V_{0} \subseteq X_{r+1} \subseteq X_{r}$.

Recall that $\operatorname{deg} Z_{j} \leq \theta_{0}<D_{r+1}$. Then

$$
\delta\left(X_{r+1}\right) \leq \max \left\{\delta\left(X_{r}\right), D_{r+1}\right\} \leq \max \left\{D_{r}, D_{r+1}\right\}=D_{r+1} .
$$

We decompose

$$
X_{r+1}=W_{1}^{\prime} \cup \cdots \cup W_{s}^{\prime} \cup W_{s+1}^{\prime} \cup \cdots \cup W_{t}^{\prime},
$$

where $W_{j}^{\prime}=W_{j} \cap Z_{1} \cap \cdots \cap Z_{s}$.

Let $j \in\{1, \ldots, s\}$. Since $W_{j} \nsubseteq Z_{j}$, every irreducible component of $W_{j}^{\prime}$ has codimension at least $\operatorname{codim}\left(W_{j}\right)+1 \geq r+1+k_{1}$.

Let $j \in\{s+1, \ldots, t\}$. Since $V_{0} \nsubseteq W_{j}$, the variety $V_{0}$ is not contained in any irreducible component of $W_{j}^{\prime}$.

We conclude that $X_{r+1}$ satisfies our claim for $r+1$.

We already mentioned in Section 2 that Theorem 2.2 gives an improved and explicit version of [AD1, Theorem 1.4] (see Corollary 2.3) and of [AD2, Theorem 1.5] (see Corollary 2.4). Theorem 2.2 has other interesting applications. For instance, we have the following. 
COROLLARY 4.1

Let $V$ be an irreducible variety of codimension $k$ which is not a translate of a subtorus. Let

$$
\theta=\delta(V)\left(200 n^{5} \log \left(n^{2} \delta(V)\right)\right)^{2(k+1) n} .
$$

Let $B \subseteq V$ be a translate of a subtorus of dimension $\operatorname{dim}(V)-1$. If $\delta_{0}(B)>\theta$, then $B\left(\theta^{-1}\right)=\emptyset$.

Proof

We apply Theorem 2.2 with $V_{0}=B$ and $V_{1}=V$. We have $k_{0}=k+1$ and $k_{1}=k$. Thus $\left(k_{0}-k_{1}+1\right) k_{0} n=2(k+1) n$. The first conclusion of Theorem 2.2 cannot hold because $\delta_{0}(B)>\theta$. It follows that $B\left(\theta^{-1}\right)$ is nondense in $B$. In view of Remark 2.5(i), we deduce that $B\left(\theta^{-1}\right)$ is empty.

We further remark that Theorem 2.2 implies Theorem 2.1, up to a slightly worse remainder term. More precisely, let $V$ be a component of an intersection $X$ of hypersurfaces of degree at most $\delta_{0}(V)$. Apply Theorem 2.2 with $V_{0}=V$ and $V_{1}=X$. Note that $V \subseteq B \subseteq X$ cannot occur. This would imply $V=B$ because $V$ is a component of $X$, contradicting the assumption in Theorem 2.1 that $V$ is not a translate of a subtorus.

\section{Quantitative description of the small points}

In this section we prove our main theorem Theorem 1.2. We then show some of its consequences.

THEOREM 1.2

Let $V \subsetneq \mathbb{G}_{\mathrm{m}}^{n}$ be a variety of codimension $k$. We decompose $V$ as a (reduced) union $X_{k} \cup \cdots \cup X_{n}$, where $X_{j}$ is an equidimensional variety of codimension $j$. We define

$$
\theta=\theta(V)=\delta(V)\left(200 n^{5} \log \left(n^{2} \delta(V)\right)\right)^{(n-k) n(n-1)} .
$$

Then

$$
\overline{V\left(\theta^{-1}\right)}=G_{k} \cup \cdots \cup G_{n},
$$

where $G_{j}$ is either the empty set or a finite union of translates $B_{j, i}$ of subtori of codimension $j$ such that $\delta_{0}\left(B_{j, i}\right) \leq \theta$. Moreover, for $r=k, \ldots, n$,

$$
\sum_{i=k}^{r} \theta^{r-i} \operatorname{deg} G_{i} \leq \sum_{i=k}^{r} \theta^{r-i} \operatorname{deg} X_{i} \leq \theta^{r} .
$$


Proof

We recall that, by our convention, the empty set is an equidimensional variety of any codimension and degree zero. Using an inductive process, we are going to construct $G_{k}, \ldots, G_{n}$ satisfying the condition of the theorem. Let $r \in\{k, \ldots, n\}$. The following claim is the inductive step of the proof.

CLAIM

There exist equidimensional varieties $G_{k}, \ldots, G_{r-1}, X_{r}^{\prime}$ of codimension $k, \ldots, r-$ $1, r$ such that

(i) for $k \leq j \leq r-1$, the variety $G_{j}$ is a finite (possibly empty) union of translates $B_{j, i}$ of subtori such that $\delta_{0}\left(B_{j, i}\right) \leq \theta$;

(ii) $\quad V\left(\theta^{-1}\right) \subseteq G_{k} \cup \cdots \cup G_{r-1} \cup X_{r}^{\prime} \cup X_{r+1} \cup \cdots \cup X_{n}$;

(iii) $\quad \sum_{i=k}^{r-1} \theta^{r-i} \operatorname{deg} G_{i}+\operatorname{deg} X_{r}^{\prime} \leq \sum_{i=k}^{r} \theta^{r-i} \operatorname{deg} X_{i}$.

In addition, $G_{r}$ is a union of components of $X_{r}^{\prime}$ for $r=k, \ldots, n$.

First, we clarify how this claim implies Theorem 1.2. Note that an equidimensional variety of codimension $n$ is a finite set of points, and points are translates of subtori. In addition, $\delta_{0}$ of a point is $1 \leq \theta$. Thus, we can define $G_{n}=X_{n}^{\prime}$. Then assertion (ii) of our claim for $r=n$ implies that

$$
V\left(\theta^{-1}\right) \subseteq G_{k} \cup \cdots \cup G_{n} .
$$

By Remark 2.5(ii), we can assume

$$
\overline{V\left(\theta^{-1}\right)}=G_{k} \cup \cdots \cup G_{n} .
$$

Since $G_{r}$ is a union of components of $X_{r}^{\prime}$, assertion (ii) of our claim for $r=k, \ldots, n$ gives the first inequality of (5.20). Corollary 5 of [P2] (with $m=n$ and $S=\mathbb{P}^{n}$ ) shows that for $\theta \geq \delta(V)$ we have

$$
\sum_{i=k}^{r} \theta^{r-i} \operatorname{deg} X_{i} \leq \theta^{r},
$$

which gives the second inequality of (5.20).

It remains to prove our claim for $r=k, \ldots, n$. We proceed by induction on $r$.

- $\quad$ For $r=k$, we simply take $X_{k}^{\prime}=X_{k}$.

- Let $r \in\{k, \ldots, n-1\}$. We first remark that if our claim holds for $r$, then it holds also with the two supplementary conditions that

(a) no component of $X_{r}^{\prime}$ is imbedded in $G_{k} \cup \cdots \cup G_{r-1}$;

(b) every component of $X_{r}^{\prime}$ meets $V\left(\theta^{-1}\right)$. 
This is clear because we can discard the components of $X_{r}^{\prime}$ not satisfying (a) or (b) without changing (ii) and (iii). Then, as an inductive hypothesis, we assume that we have constructed $G_{k}, \ldots, G_{r-1}, X_{r}^{\prime}$ satisfying our claim and the properties (a) and (b), as well.

We decompose $X_{r}^{\prime}$ as

$$
X_{r}^{\prime}=G_{r} \cup W_{1} \cup \cdots \cup W_{s},
$$

where

- $G_{r}$ is the union of the components $B_{r, i}$ of $X_{r}^{\prime}$ which are translates of subtori and such that $\delta_{0}\left(B_{r, i}\right) \leq \theta$ (possibly $G_{r}=\emptyset$ );

- $\quad W_{1}, \ldots, W_{s}$ are the components of $X_{r}^{\prime}$ not in $G_{r}$ (possibly $s=0$ ).

Clearly, the first assertion of our claim for $r+1$ is satisfied. It remains to show (ii) and (iii) for $r+1$.

Let $i \in\{1, \ldots, s\}$.

Remark. There does not exist any translate $B$ of a subtorus such that $\delta_{0}(B) \leq \theta$ and $W_{i} \subseteq B \subseteq V$.

Proof

Assume by contradiction that there exists a translate $B$ of a subtorus such that $\delta_{0}(B) \leq$ $\theta$ and $W_{i} \subseteq B \subseteq V$. By condition (b), $W_{i}\left(\theta^{-1}\right) \neq \emptyset$. Then Remark 2.5 (ii) gives $\overline{B\left(\theta^{-1}\right)}=B$. Furthermore, $B\left(\theta^{-1}\right) \subseteq V\left(\theta^{-1}\right)$ and $\operatorname{dim} B \geq r$. Thus

$$
W_{i} \subseteq B \subseteq G_{k} \cup \cdots \cup G_{r},
$$

contradicting either (a) or the definition of $G_{r}$.

We now apply Theorem 2.2 to the varieties $V_{0}=W_{i}$ and $V_{1}=V$. We have $k_{0}=$ $r \leq n-1$ and $k_{1}=k$. The first conclusion of Theorem 2.2 cannot occur because of the previous remark. Thus, the second conclusion must hold. Namely, there exists a hypersurface $Z_{i}$ of degree at most $\theta$ such that $W_{i} \nsubseteq Z_{i}$ and $W_{i}\left(\theta^{-1}\right) \subseteq Z_{i}$. By Krull's Hauptsatz, $W_{i} \cap Z_{i}$ is either the empty set or it is an equidimensional variety of codimension $r+1$.

We define

$$
X_{r+1}^{\prime}=X_{r+1} \cup \bigcup_{i=1}^{s}\left(W_{i} \cap Z_{i}\right) .
$$

By construction,

$$
V\left(\theta^{-1}\right) \subseteq G_{k} \cup \cdots \cup G_{r} \cup X_{r+1}^{\prime} \cup X_{r+2} \cup \cdots \cup X_{n} .
$$

Then (ii) of our claim is satisfied for $r+1$. 
By Bézout's theorem, by the definition of $X_{r+1}^{\prime}$, and by $\operatorname{deg} Z_{i} \leq \theta$, we deduce

$$
\operatorname{deg} X_{r+1}^{\prime} \leq \theta\left(\sum_{i=1}^{s} \operatorname{deg} W_{i}\right)+\operatorname{deg} X_{r+1}
$$

Substituting $\sum_{i=1}^{s} \operatorname{deg} W_{i}=\operatorname{deg} X_{r}^{\prime}-\operatorname{deg} G_{r}$ (which rises directly from (5.21)), we obtain

$$
\operatorname{deg} X_{r+1}^{\prime} \leq \theta\left(\operatorname{deg} X_{r}^{\prime}-\operatorname{deg} G_{r}\right)+\operatorname{deg} X_{r+1} .
$$

Thus

$$
\begin{aligned}
\sum_{i=k}^{r} \theta^{r+1-i} \operatorname{deg} G_{i}+\operatorname{deg} X_{r+1}^{\prime} \leq & \sum_{i=k}^{r} \theta^{r+1-i} \operatorname{deg} G_{i} \\
& +\theta\left(\operatorname{deg} X_{r}^{\prime}-\operatorname{deg} G_{r}\right)+\operatorname{deg} X_{r+1} \\
= & \theta\left(\sum_{i=k}^{r-1} \theta^{r-i} \operatorname{deg} G_{i}+\operatorname{deg} X_{r}^{\prime}\right)+\operatorname{deg} X_{r+1} .
\end{aligned}
$$

By the inductive hypothesis, $G_{k}, \ldots, G_{r-1}, X_{r}^{\prime}$ satisfy (iii) of our claim:

$$
\sum_{i=k}^{r-1} \theta^{r-i} \operatorname{deg} G_{i}+\operatorname{deg} X_{r}^{\prime} \leq \sum_{i=k}^{r} \theta^{r-i} \operatorname{deg} X_{i} .
$$

Hence

$$
\theta\left(\sum_{i=k}^{r-1} \theta^{r-i} \operatorname{deg} G_{i}+\operatorname{deg} X_{r}^{\prime}\right)+\operatorname{deg} X_{r+1} \leq \sum_{i=k}^{r+1} \theta^{r+1-i} \operatorname{deg} X_{i} .
$$

This proves (iii) of our claim for $r+1$.

Proof of Corollary 1.3

Obviously, for all varieties $V \subseteq W$ and real numbers $\varepsilon \geq 0$, it holds that $V(\varepsilon)=V \cap W(\varepsilon)$. Applying theorem 1.2 to $W$, we immediately obtain $V\left(\theta(W)^{-1}\right) \subseteq V \cap \bigcup B_{j}$, where $B_{j} \subseteq W$ are translates of subtori such that $\sum \operatorname{deg} B_{j} \leq \theta(W)^{n}$ and $\delta_{0}\left(B_{j}\right) \leq \theta(W)$. Consequently, if $V\left(\theta(W)^{-1}\right)$ is dense in $V$, then $V \subseteq \bigcup B_{j}$ and each component of $V$ is contained in a translate $B_{j}$ of a subtorus with $\delta_{0}\left(B_{j}\right) \leq \theta(W)$. 
COROLLARY 5.1

Let $V$ be an irreducible subvariety of $\mathbb{G}_{\mathrm{m}}^{n}$ which is not a translate of a subtorus. Define

$$
\theta_{0}=\delta_{0}(V)\left(200 n^{5} \log \left(n^{2} \delta_{0}(V)\right)\right)^{n(n-1)^{2}} .
$$

Then $V\left(\theta_{0}^{-1}\right)$ is contained in a finite union of translates $B_{j}$ of proper subtori such that $V \nsubseteq B_{j}, \delta_{0}\left(B_{j}\right) \leq \theta_{0}$, and $\sum_{j} \operatorname{deg}\left(B_{j}\right) \leq \theta_{0}^{n}$.

Proof

Apply Corollary 1.3 with $W$ an intersection of hypersurfaces of degree at most $\delta_{0}(V)$ such that $V$ is a component of $W$. Then $\theta(W) \leq \theta_{0}$, and the claim is proved except for the assertion $V \nsubseteq B_{j}$. Note that, if $V \subseteq B_{j} \subseteq W$, then $V=B_{j}$ because $V$ is a component in $W$. This contradicts the assumption that $V$ is not the translate of a subtorus.

COROLLARY 5.2

Let $V$ be an irreducible subvariety of $\mathbb{G}_{\mathrm{m}}^{n}$. Let

$$
\theta_{\omega}=\omega(V)\left(200 n^{5} \log \left(n^{2} \omega(V)\right)\right)^{n(n-1)^{2}} .
$$

Then $V\left(\theta_{\omega}^{-1}\right)$ is contained in a finite union of translates $B_{j}$ of proper subtori such that $\delta_{0}\left(B_{j}\right) \leq \theta_{\omega}$ and $\sum_{j} \operatorname{deg}\left(B_{j}\right) \leq \theta_{\omega}^{n}$.

Proof

Apply Corollary 1.3 with $W$ a hypersurface such that $V \subseteq W$ and $\operatorname{deg}(W)=\omega(V)$. Such a $W$ exists by definition of $\omega$.

Note that Corollary 5.2 immediately implies that, for $V$ transverse, $\hat{\mu}^{\text {ess }}(V) \geq \theta_{\omega}^{-1}$.

We further remark that the bound (5.20) of Theorem 1.2 can be slightly improved for an irreducible $V$ of codimension $k$ which is not a translate of a subtorus. Indeed, by Theorem 2.1, there exists a hypersurface $Z$ with

$$
\operatorname{deg} Z \leq \theta_{0}=\delta_{0}(V)\left(27 n^{2} \log \left(n^{2} \delta_{0}(V)\right)\right)^{k n},
$$

which does not contain $V$ and such that $V\left(\theta_{0}^{-1}\right) \subseteq V \cap Z$. Then $\operatorname{deg}(V \cap Z) \leq$ $\theta_{0} \operatorname{deg}(V), \operatorname{codim}(V \cap Z)=k+1$, and $\delta(V \cap Z) \leq \max \left(\delta(V), \theta_{0}\right)$. Thus $\theta(V \cap Z)$ is essentially bounded by $\theta(V)$. Theorem 1.2 applied to the equidimensional variety $V \cap Z$ gives a sharper version of the bound (5.20) obtained applying Theorem 1.2 directly to the variety $V$ : substantially the bound $\theta(V)^{r}$ is replaced by $\theta_{0} \theta(V)^{r-1}$. 
In this spirit, one can play on Theorem 1.2 producing a series of essentially similar corollaries.

An example (inspired by [AD2, p. 555]) clarifies the situation. Let $m \geq 3$ be an integer. In $\mathbb{G}_{\mathrm{m}}^{4}$, we consider the hypersurfaces

$$
Z_{m}=\left\{x^{m}+y^{m}-1=0\right\}, \quad W=\left\{x^{2}+x^{3}-z-t=0\right\},
$$

the variety $V_{m}=Z_{m} \cap W$, the subtori of $W$,

$$
T_{1}=\left\{z=x^{2}, t=x^{3}\right\}, \quad T_{2}=\left\{z=x^{3}, t=x^{2}\right\},
$$

and the curves $C_{m, i}=V_{m} \cap T_{i}=Z_{m} \cap T_{i}$.

The varieties $V_{m}, W$, and $Z_{m}$ are transverse, while $C_{m, i}$ is contained in $T_{i}$ and $\delta_{0}\left(T_{i}\right)=3$. Moreover, $\omega\left(V_{m}\right)=\operatorname{deg}(W)=3, \delta\left(V_{m}\right)=\operatorname{deg}\left(Z_{m}\right)=m, \omega\left(C_{m, i}\right)=2$, $\delta\left(C_{m, i}\right)=\operatorname{deg}\left(Z_{m}\right)=m$.

The points are

$$
P_{m, n}^{1}=\left(2^{1 / n},\left(1-2^{m / n}\right)^{1 / m}, 2^{3 / n}, 2^{2 / n}\right) \in C_{m, 1}
$$

and

$$
P_{m, n}^{2}=\left(2^{1 / n},\left(1-2^{m / n}\right)^{1 / m}, 2^{2 / n}, 2^{3 / n}\right) \in C_{m, 2} .
$$

For $n$ large, we have $0 \leq c^{\prime} / m \leq h\left(P_{m, n}^{i}\right) \leq c / m$ for some absolute positive constants $c$ and $c^{\prime}$ independent of $m$. Thus $\hat{\mu}^{\text {ess }}\left(C_{m, i}\right) \leq c / m$. This shows that the first conclusion of Theorem 2.2 cannot be avoided. More precisely, let $f$ be any positive real function. Then we cannot expect $\hat{\mu}^{\text {ess }}\left(V_{0}\right) \geq f\left(\delta\left(V_{1}\right)\right)$ for $V_{0}$ contained in a translate of a subtorus $\subseteq V_{1}$ of small $\delta_{0}$. This was contradicted by choosing $V_{1}=W$ and $V_{0}=C_{m, i}$ for $m$ large enough.

As remarked in [AD2], we cannot "replace $\delta(V)$ by $\omega(V)$ " in Theorem 1.2. More precisely, let $f$ be any positive real function. Then there exists a positive integer $m^{\prime}$ such that $c / m^{\prime}<f(3)$. Thus, for any sufficiently large $n$, the points $P_{m^{\prime}, n}^{1}$ and $P_{m^{\prime}, n}^{2}$ lie on $V_{m^{\prime}}\left(f\left(\omega\left(V_{m^{\prime}}\right)\right)\right)$. Recall that $V^{0}$ is the complement in $V$ of the union of all translates of subtori of positive dimension contained in $V$. Since $V$ does not contain any translate of positive dimension, $V_{m^{\prime}}^{0}=V_{m^{\prime}}$. It follows that the set $V_{m^{\prime}}^{0}\left(f\left(\omega\left(V_{m^{\prime}}\right)\right)\right)$ is not finite.

Let $V$ be a subvariety of $\mathbb{G}_{\mathrm{m}}^{n}$. Notice that $V(0)$ is the set of torsion points of $V$. By the toric version of the Manin-Mumford conjecture (see [L]),

$$
\overline{V(0)}=B_{1} \cup \cdots \cup B_{t}
$$


with $B_{j} \subseteq V$ torsion varieties. We recall that $V^{u}$ is the union of all torsion varieties contained in $V$. Since the torsion is dense in a torsion variety,

$$
V^{u}=\overline{V(0)}
$$

We say that a torsion variety $B$ is maximal in $V$ if $B \subseteq V$ and $B$ is not strictly contained in any translate $B^{\prime} \subseteq V$ of a subtorus. If a translate $B^{\prime}$ contains a torsion variety, then $B^{\prime}$ is itself a torsion variety. Thus, discarding torsion varieties contained in others, we can assume that $B_{1}, \ldots, B_{t}$ are precisely the maximal torsion varieties of $V$ and

$$
V^{u}=B_{1} \cup \cdots \cup B_{t} .
$$

The following corollary improves the known upper bounds on $t$ quoted in the introduction.

COROLLARY 5.3

Let $V$ be a subvariety of $\subseteq \mathbb{G}_{\mathrm{m}}^{n}$ of codimension $k$. Let

$$
\theta(V)=\delta(V)\left(200 n^{5} \log \left(n^{2} \delta(V)\right)\right)^{(n-k) n(n-1)}
$$

be as in (1.5). Let $B_{1}, \ldots, B_{t}$ be the maximal torsion varieties of $V$. Then $\delta_{0}\left(B_{j}\right) \leq$ $\theta(V)$ and

$$
\sum_{j=1}^{t} \theta(V)^{\operatorname{dim}\left(B_{j}\right)} \operatorname{deg}\left(B_{j}\right) \leq \theta(V)^{n} .
$$

In particular, $t \leq \theta(V)^{n}$.

Proof

The discussion above shows that

$$
B_{1} \cup \cdots \cup B_{t}=\overline{V(0)}=V^{u} .
$$

Let $\theta=\theta(V)$. Since $V(0) \subseteq V\left(\theta^{-1}\right)$, Theorem 1.2 gives

$$
B_{1} \cup \cdots \cup B_{t}=\overline{V(0)} \subseteq \overline{V\left(\theta^{-1}\right)}=B_{1}^{\prime} \cup \cdots \cup B_{t^{\prime}}^{\prime},
$$

where $B_{j}^{\prime} \subseteq V$ are translates of subtori satisfying $\delta_{0}\left(B_{j}^{\prime}\right) \leq \theta$ and

$$
\sum_{j=1}^{t^{\prime}} \theta^{\operatorname{dim}\left(B_{j}^{\prime}\right)} \operatorname{deg}\left(B_{j}^{\prime}\right) \leq \theta^{n} .
$$

The $B_{j}$ are maximal; thus $\left\{B_{1}, \ldots, B_{t}\right\} \subseteq\left\{B_{1}^{\prime}, \ldots, B_{t^{\prime}}^{\prime}\right\}$. 


\section{Applications to the Mordell-Lang plus Bogomolov problem}

We first prove Corollary 1.4. Let us recall the statement.

COROLLARY 1.4

Let $\Gamma$ be a subgroup of $\mathbb{G}_{\mathrm{m}}^{n}$ of finite rank $r$, and let $V \subsetneq \mathbb{G}_{\mathrm{m}}^{n}$ be a subvariety of codimension $k$. As in (1.5), let

$$
\theta(V)=\delta(V)\left(200 n^{5} \log \left(n^{2} \delta(V)\right)\right)^{(n-k) n(n-1)} .
$$

Then for $C \geq 1$ and for any nonnegative $\varepsilon \leq(2 \theta(V))^{-1}$,

$$
\left|V^{0}(C) \cap \Gamma_{\varepsilon}\right| \leq(5 n C)^{r} \theta(V)^{n+r} .
$$

Proof

For $\boldsymbol{\alpha} \in \mathbb{G}_{\mathrm{m}}^{n}$, let

$$
h_{s}(\boldsymbol{\alpha})=h\left(\alpha_{1}\right)+\cdots+h\left(\alpha_{n}\right)
$$

be the height on $\mathbb{G}_{\mathrm{m}}^{n}$ with respect to $\mathbb{G}_{\mathrm{m}}^{n} \subset\left(\mathbb{P}^{1}\right)^{n}$. Let $\rho \geq 0$ and $\mu>0$ such that $\rho / 2 \mu \geq \varepsilon$. Since $\Gamma$ has finite rank $r$, by [R, Lemma 2.1], there exists a finite subset $E$ of $\Gamma$ of cardinality at most $(4 \mu+3)^{r}$ such that

$$
\left\{\mathbf{x} \in \Gamma_{\varepsilon}: h_{s}(\mathbf{x}) \leq \rho\right\} \subseteq \bigcup_{\mathbf{y} \in E}\left\{\mathbf{x} \in \Gamma_{\varepsilon}: h_{s}\left(\mathbf{x y}^{-1}\right) \leq \frac{\rho}{\mu}\right\} .
$$

Since

$$
h \leq h_{s} \leq n h,
$$

this implies

$$
\left|V^{0}\left(n^{-1} \rho\right) \cap \Gamma_{\varepsilon}\right| \leq \sum_{\mathbf{y} \in E}\left|\left(\mathbf{y}^{-1} V\right)^{0}\left(\frac{\rho}{\mu}\right)\right| .
$$

Let $\theta=\theta(V)$. We choose $\rho=n C$ and $\mu=n C \theta$. We have $\rho / 2 \mu=(2 \theta)^{-1} \geq \varepsilon$. By Theorem 1.2, with $V$ replaced by $\mathbf{y}^{-1} V$, we deduce

$$
\left|\left(\mathbf{y}^{-1} V\right)^{0}\left(\frac{\rho}{\mu}\right)\right| \leq \theta^{n} .
$$

In view of (6.22),

$$
\left|V^{0}(C) \cap \Gamma_{\varepsilon}\right| \leq|E| \theta^{n} \leq(4 n C \theta+3)^{r} \theta^{n} .
$$

We finally remark that $4 n C \theta+3 \leq 5 n C \theta$ since $3 \leq n \theta \leq n C \theta$. 
Given a subset $\Gamma \subseteq \mathbb{G}_{\mathrm{m}}^{n}$ and $\varepsilon \geq 0$, we consider the conic neighborhood

$$
\mathscr{C}(\Gamma, \varepsilon)=\left\{\boldsymbol{\alpha} \in \mathbb{G}_{\mathrm{m}}^{n}: \boldsymbol{\alpha}=\mathbf{x z} \text { with } \mathbf{x} \in \Gamma \text { and } h(\mathbf{z}) \leq(1+h(\mathbf{x})) \varepsilon\right\} .
$$

Let $\varepsilon=n^{-1} \exp \left\{-(4 n)^{3 n}\right\}$, and let $\Gamma \subseteq \mathbb{G}_{\mathrm{m}}^{n}$ be a subgroup of rank $r$. In [ESS, Theorem 2.1], the authors show that the set of $\alpha \in \mathbb{G}_{\mathrm{m}}^{n}$ satisfying

$$
\alpha_{1}+\cdots+\alpha_{n}=1, \quad \boldsymbol{\alpha} \in \mathscr{C}(\Gamma, \varepsilon)
$$

is contained in the union of at most $\exp \left\{(5 n)^{3 n}(r+1)\right\}$ proper linear subspaces of $\overline{\mathbb{Q}}^{n}$. Corollary 1.4 allows us to save an exponential in these estimates.

THEOREM 6.1

Let $\varepsilon=(8 n)^{-6 n^{3}}$. Then the set of $\boldsymbol{\alpha} \in \mathbb{G}_{\mathrm{m}}^{n}$ satisfying (6.23) is contained in the union of at most $(8 n)^{6 n^{3}(n+r)}$ proper linear subspaces of $\overline{\mathbb{Q}}^{n}$.

Proof

We follow [ESS]. By the reduction process of [ESS, Section 6], it is sufficient to bound the number of proper linear subspaces containing the solutions $\alpha$ of $\alpha_{1}+\cdots+\alpha_{n}=1$ such that

$$
\alpha \in \mathscr{C}(\Gamma, \varepsilon) \cap \mathbb{G}_{\mathrm{m}}^{n}(F)
$$

We decompose

$$
\boldsymbol{\alpha}=\mathbf{x z} \quad \text { with } \mathbf{x} \in \Gamma, \mathbf{z} \in \mathbb{G}_{\mathrm{m}}^{n}(F), h(\mathbf{z}) \leq(1+h(\mathbf{x})) \varepsilon,
$$

where $F$ is a fixed number field. As in [ESS], we say that a solution is "large" (see [ESS, (9.1)]) if $h(\mathbf{x})>4 n \log n$. The argument of [ESS, Sections 8, 9, 10] shows* that the number of large solutions is contained in at most

$$
A=2^{2(2 n+9)^{2}}\left(8 n^{2}+2 n\right)^{n+4+r}
$$

proper linear subspaces of $F^{n}$. We have

$$
A \leq \frac{1}{4}(8 n)^{6 n^{3}(n+r)}
$$

*Indeed, in these sections, the value of $\varepsilon$ is used only to guarantee [ESS, (9.19)]. This equation still holds for our choice of $\varepsilon$ since $h(\mathbf{z}) \leq \varepsilon(1+h(\mathbf{x}))=(8 n)^{-6 n^{3}}(1+h(\mathbf{x})) \leq h(\mathbf{x}) /(8 n)$ if $h(\mathbf{x}) \geq 1$. 
Indeed, using $8 n^{2}+2 n \leq 2^{4} n^{2}$, we obtain $A /(1 / 4)(8 n)^{6 n^{3}(n+r)} \leq 2^{a} n^{b}$ with

$$
\begin{aligned}
& a=2(2 n+9)^{2}+4(4+n+r)+2-18 n^{3}(n+r), \\
& b=2(4+n+r)-6 n^{3}(n+r) .
\end{aligned}
$$

Since $b<0$ and $a+b<0$, we have $2^{a} n^{b} \leq 2^{a+b}<1$.

We now consider "small" solutions $\boldsymbol{\alpha}=\mathbf{x z}$ satisfying $h(\mathbf{x}) \leq 4 n \log n$. Let $V$ be the subvariety of $\mathbb{G}_{\mathrm{m}}^{n}$ defined by $\alpha_{1}+\cdots+\alpha_{n}=1$. Then $\delta(V)=1$ and

$$
\theta(V)=\left(400 n^{5} \log n\right)^{n(n-1)^{2}} .
$$

We have $400 n^{5} \log n \leq 400 n^{11 / 2} \leq(1 / 4)(8 n)^{11 / 2}$ and

$$
\theta(V) \leq \frac{1}{4}(8 n)^{(11 / 2) n^{3}} .
$$

By [S, p. 161], $V^{0}$ is the set of nondegenerate solutions of this equation. Moreover, small solutions satisfy

$$
\begin{aligned}
h(\boldsymbol{\alpha}) \leq h(\mathbf{x})+h(\mathbf{z}) & \leq 4 n \log n+(1+4 n \log n) \varepsilon \\
& \leq(4+5 \varepsilon) n \log n \\
& \leq 5 n^{2}
\end{aligned}
$$

and

$$
h(\mathbf{z}) \leq(1+4 n \log n) \varepsilon .
$$

Note that

$$
(1+4 n \log n) \varepsilon \cdot 2 \theta(V) \leq 5 n^{2}(8 n)^{-6 n^{3}}(8 n)^{(11 / 2) n^{3}}<1 .
$$

Thus we can apply Corollary 1.4 with $C=5 n^{2}$. Using the inequality $5 n C \leq(8 n)^{3}$, we find that there are at most

$$
B=(5 n C)^{r} \theta(V)^{n+r} \leq \frac{1}{4}(8 n)^{3 r+(11 / 2) n^{3}(n+r)} \leq \frac{1}{4}(8 n)^{6 n^{3}(n+r)}
$$

nondegenerate small solutions. Since the degenerate solutions are contained in the union of at most $2^{n}$ proper linear subspaces, to cover the set of all solutions we need at most

$$
A+B+2^{n} \leq \frac{1}{4}(8 n)^{6 n^{3}(n+r)}+\frac{1}{4}(8 n)^{6 n^{3}(n+r)}+2^{n} \leq(8 n)^{6 n^{3}(n+r)}
$$

subspaces. 
Using this last theorem, we deduce the following.

THEOREM 6.2

Let $K$ be an algebraically closed field of characteristic zero. Let $\left(a_{1}, \ldots, a_{n}\right) \in$ $\mathbb{G}_{\mathrm{m}}^{n}(K)$, and let $\Gamma$ be a subgroup of $\mathbb{G}_{\mathrm{m}}^{n}(K)$ of finite rank $r$. Then the equation

$$
a_{1} \alpha_{1}+a_{2} \alpha_{2}+\cdots+a_{n} \alpha_{n}=1 \quad \text { with } \alpha \in \Gamma
$$

has at most $A(n, r)=(8 n)^{4 n^{4}(n+r+1)}$ nondegenerate solutions.

Proof

By [ESS, Lemma 3.2], we may suppose $K=\overline{\mathbb{Q}}$. Let $A^{\prime}(n, r)$ be the number of nondegenerate solutions of (6.24). We prove by induction on $n$ that $A^{\prime}(n, r) \leq A(n, r)$ for every positive integer $r$. Our claim is obvious if $n=1$. Let $n$ be an integer at least 2 , and assume $A^{\prime}(m, r) \leq A(m, r)$ for $1 \leq m<n$ and for every positive integer $r$. Let $B(n, r)=(8 n)^{6 n^{3}(n+r)}$ be the bound of Theorem 6.1. Then, by the arguments of [ESS, Section 4] and by the inductive hypothesis, ${ }^{*}$

$$
A^{\prime}(n, r) \leq 2^{n} A(n-1, r) B(n, r+1) \leq(8 n)^{c}
$$

with

$$
\begin{aligned}
c & =n+4(n-1)^{4}(n+r)+6 n^{3}(n+r+1) \\
& \leq\left(1+4(n-1)^{4}+6 n^{3}\right)(n+r+1) \\
& \leq 4 n^{4}(n+r+1) .
\end{aligned}
$$

Thus $A^{\prime}(n, r) \leq(8 n)^{4 n^{4}(n+r+1)}$, as required.

As mentioned, Theorem 6.2 has an application to estimate for the multiplicities in a linear recurrence sequence $\left\{u_{m}\right\}_{m \in \mathbb{Z}}$ of order $n \geq 1$ with elements in $K$, for $K$ an algebraically closed field of characteristic zero. Let $\left\{u_{m}\right\}$ be such a sequence. Then it satisfies a minimal relation

$$
u_{m+n}=c_{1} u_{m+n-1}+\cdots+c_{n} u_{m} \quad(m \in \mathbb{Z})
$$

with $c_{1}, \ldots, c_{n} \in K$. We say that $\left\{u_{m}\right\}$ is simple if its companion polynomial $G(z)=$ $z^{n}-c_{1} z^{n-1}-\cdots-c_{n}$ has only simple roots. Let

$$
\mathscr{S}\left(u_{m}\right)=\left\{k: u_{k}=0\right\} .
$$

*Remark that, for integers $a, b \geq 1$ and $r_{1}, r_{2} \geq 0$, the function $A(n, r)$ satisfies the inequality $A\left(a, r_{1}\right) A\left(b, r_{2}\right) \leq$ $A\left(a+b-1, r_{1}+r_{2}\right)$. Thus [ESS, inequality (4.12)] still holds. 
The Skolem-Mahler-Lech theorem asserts that for an arbitrary linear recurrence sequence $\left\{u_{m}\right\}$ of order $n \geq 1$ the set $\mathscr{S}\left(u_{m}\right)$ is a finite union of arithmetic progressions (where single elements of $\mathbb{Z}$ are trivial arithmetic progressions). The following corollary improves of one exponential the bounds of [ESS, Theorem 1.2] on the Skolem-Mahler-Lech theorem.

COROLLARY 6.3

Let $\left\{u_{m}\right\}$ be a simple linear recurrence sequence in $K$ of order $n \geq 1$. Then $\mathscr{S}\left(u_{m}\right)$ is the union of at most $(8 n)^{4 n^{5}}$ arithmetic progressions.

Proof

We follow closely the inductive proof of [ESS, Theorem 1.2] in [ESS, Section 5]. We define $W(n)=(8 n)^{4 n^{5}}$. Using our Theorem 6.2 instead of [ESS, Theorem 1.2], we see that $[\mathrm{ESS},(5.3)]$ has at most

$$
A(n-1,1)=(8(n-1))^{4(n-1)^{4}(n+1)} \leq \frac{1}{2}(8 n)^{4 n^{5}}
$$

nondegenerate solutions. For $2 \leq l \leq n-2$, we have

$$
W(l) W(n-l) \leq(8 n)^{4 n^{5}-n}
$$

because $l^{5}+(n-l)^{5} \leq(l+(n-l)) \max (l, n-l)^{4} \leq n(n-2)^{4} \leq n^{5}-n$. Thus

$$
A(n-1,1)+2^{n} \max _{2 \leq l \leq n-2} W(l) W(n-l) \leq \frac{1}{2}(8 n)^{4 n^{5}}+\frac{1}{2}(8 n)^{4 n^{5}}=W(n) .
$$

As in [ESS], we conclude that our result holds.

Acknowledgments. We thank the referees for their remarks and valuable suggestions. We express our gratitude to Patrice Philippon and Martín Sombra for numerous helpful conversations. We are indebted to Jan-Hendrik Evertse for suggesting to us the application of our result to the estimate of nondegenerate solutions of a linear equation in a group of finite rank. We kindly thank Corentin Pontreau and Gaël Rémond for reading a preliminary version of this article.

\section{References}


[A] F. AMOROSO, Bogomolov on tori revisited, preprint, 2007, http://hal.archives-ouvertes.fr/hal-00132119

[AD1] F. AMOROSO and S. DAVID, Minoration de la hauteur normalisée dans un tore, J. Inst. Math. Jussieu 2 (2003), 335-381. MR 1990219

[AD2] Points de petite hauteur sur une sous-variété d'un tore, Compos. Math. 142 (2006), 551-562. MR 2231192

[BMZ] E. BOMBIERI, D. MASSER, and U. ZANNIER, Intersecting a curve with algebraic subgroups of multiplicative groups, Internat. Math. Res. Notices 1999, no. 20, 1119-1140. MR 1728021

[BZ] E. BOMBIERI and U. ZANNIER, Algebraic points on subvarieties of $\mathbb{G}_{\mathrm{m}}^{n}$, Internat. Math. Res. Notices 1995, no. 7, 333-347. MR 1350686

[C] M. CHARDIN, Une majoration de la fonction de Hilbert et ses conséquences pour l’interpolation algébrique, Bull. Soc. Math. France 117 (1988), 305-318. MR 1020108

[CP] M. CHARDIN and P. PHILIPPON, Régularité et interpolation, J. Algebraic Geom. 8 (1999), 471-481; Erratum, J. Algebraic Geom. 11 (2002), 599-600. MR 1689351; MR 1894934

[DP] S. DAVID and P. PHILIPPON, Minorations des hauteurs normalisées des sous-variétés des tores, Ann. Scuola Norm. Sup. Pisa Cl. Sci. (4) 28 (1999), 489-543; Errata, Ann. Scuola Norm. Sup. Pisa Cl. Sci. (4) 29 (2000), 729 - 731. MR 1736526; MR 1817716

[E] J.-H. EVERTSE, "Points on subvarieties of tori" In A Panorama of Number Theory or the View from Baker's Garden (Zürich, 1999), Cambridge Univ. Press, Cambridge, 2002, 214-230. MR 1975454

[ESS] J.-H. EVERTSE, H.-P. SCHLICKEWEI, and W. M. SCHMIDT, Linear equations in variables which lie in a multiplicative group, Ann. of Math. (2) 155 (2002), 807-836. MR 1923966

[H] P. HABEGGER, A Bogomolov property for curves modulo algebraic subgroups, Bull. Soc. Math. France 137 (2009), 93 - 125. MR 2496702

[Hi] M. HINDRY, Autour d'une conjecture de Serge Lang, Invent. Math. 94 (1988), 575 - 603. MR 0969244

[L] M. LAURENT, Équations diophantiennes exponentielles, Invent. Math. 78 (1984), 299-327. MR 0767195

[P1] P. PHILIPPON, Lemmes de zéros dans les groupes algébriques commutatifs, Bull. Soc. Math. France 114 (1986), 353-383. MR 0878242

[P2] - Sur des hauteurs alternatives, III, J. Math. Pures Appl. (9) 74 (1995), 345-365. MR 1341770

[Po] B. POONEN, Mordell-Lang plus Bogomolov, Invent. Math. 137 (1999), 413 - 425. MR 1705838

[R] G. RÉMOND, Sur les sous-variétés des tores, Compositio Math. 134 (2002), 337-366. MR 1943907

[RS] J. B. ROSSER and L. SCHOENFELD, Approximate formulas for some functions of prime numbers, Illinois J. Math. 6 (1962), 64-94. MR 0137689 
[S] W. M. SCHMIDT, "Heights of points on subvarieties of $\mathbb{G}_{\mathrm{m}}^{n}$ " in Number Theory (Paris, 1993 - 1994), London Math. Soc. Lecture Note Ser. 235, Cambridge Univ. Press, Cambridge 1996, 157-187. MR 1628798

[V] E. VIADA, The intersection of a curve with a union of translated codimension-two subgroups in a power of an elliptic curve, Algebra Number Theory 2 (2008), 249-298. MR 2407116

Amoroso

Laboratoire de mathématiques Nicolas Oresme, CNRS UMR 6139, Université de Caen,

F-14032 Caen CEDEX, France; amoroso@math.unicaen.fr

Viada

Department of Mathematics, Université de Fribourg, CH-1700 Fribourg Pérolles, Switzerland; evelina.viada@unifr.ch 